\title{
Reflection and transmission of SH-waves at a corrugated interface between two laterally and vertically heterogeneous anisotropic elastic solid half-spaces
}

\author{
S. K. Tomar ${ }^{1}$ and Jatinder Kaur ${ }^{2}$ \\ ${ }^{1}$ Department of Mathematics, Panjab University, Chandigarh-160 014, India \\ ${ }^{2}$ Department of Mathematics, Guru Nanak Girls College, Santpura, Yamuna Nagar-135 001, Haryana, India
}

(Received August 5, 2002; Revised September 25, 2003; Accepted September 26, 2003)

\begin{abstract}
This paper is concerned with the reflection and transmission coefficients of SH-waves at a corrugated interface between two anisotropic heterogeneous elastic solid half spaces. Both the half spaces are taken transversely isotropic and laterally and vertically heterogeneous. The Rayleigh's method of approximation is adopted and expressions for reflection and transmission coefficients are obtained in closed form for the first-order approximation of the corrugation. In Rayleigh's method, expressions in boundary conditions containing the function defining the corrugated boundary are expanded in Fourier series and unknown coefficients in the solutions are determined to any given order of approximation in terms of a small parameter characteristic of the boundary. The analytical expressions of these coefficients show that they depend upon corrugation of the interface and are strongly influenced by the anisotropy and heterogeneity of the half-spaces. Numerical computations are performed for the case of a particular corrugated interface: $\zeta=c \cos k^{*} x$ showing that the effect of heterogeneity on the reflection and transmission coefficients is minimum near the normal incidence and dominance of this effect increases with the angle of incidence. For incident wave striking at $45^{\circ}$, the effect of the corrugation is found significant on the reflection and transmission coefficients. The maximum effect of transverse isotropy on the reflection and transmission coefficients is observed at normal incidence when the values of the anisotropy parameters are 0.5 and 0.8 for the upper and lower half-spaces, respectively. The effect of frequency of the incident wave is observed on all reflected and refracted waves. The analytical expressions derived by Tomar and Saini (1997), Gupta (1987) and Asano (1960) are obtained as particular cases with our formulation.
\end{abstract}

Key words: Reflection, transmission, SH-wave, Rayleigh's method, heterogeneity, anisotropy, corrugation.

\section{Introduction}

Solving the problem of reflection and refraction of seismic waves from discontinuities is of great help to better understand the internal structure of the earth, and to detect valuable materials as well as hydro-carbons beneath the earth surface. Earth heterogeneities such as mountain basin, mountain roots and ore bodies affect the reflection and transmission of seismic waves, thus the study of the reflection and refraction coefficients due to rough surfaces is of great practical importance in Seismology.

A number of researchers have attempted the problem of reflection and refraction of seismic waves at corrugated interfaces using different methods. Sato (1955) investigated the reflection of elastic waves at a corrugated, free surface by using the Rayleigh's method of approximation. Asano (1960, 1961, 1966) also used the Rayleigh's method to address problems of reflection and refraction of elastic waves at a corrugated interface between two uniform elastic half spaces. Abubakar (1962a) and Dunkin and Eringen (1962) used the perturbation method to study the problem of reflection of body waves from a rough surface of a semi-infinite elastic solid. In perturbation method, the amplitudes of su-

Copy right(c) The Society of Geomagnetism and Earth, Planetary and Space Sciences (SGEPSS); The Seismological Society of Japan; The Volcanological Society of Japan The Geodetic Society of Japan; The Japanese Society for Planetary Sciences. perposed scattered plane waves are expressed into sums of terms whose order of magnitudes are proportional to the powers of the amplitude of the rough surface, the curvature of the rough surface should be small everywhere. Later, Abubakar (1962b, c), using the perturbation technique, studied the reflection and refraction of $\mathrm{SH}$-waves at an irregular interface between two uniform elastic solid half spaces. Adams and Chung-Po-Chang (1964) applied Weber integral to solve wave equation and studied the wave propagation phenomenon at an irregular infinite interface. Aki and Larner (1970) investigated the surface motion of a layered medium with irregular interface due to incident plane $\mathrm{SH}$ wave. The effect of surface irregularity on the propagation of waves in an elastic plate was studied by Sumner and Deresiewicz (1972) using the perturbation technique. Some notable references pertaining to the subject of waves on irregular boundaries are Gilbert and Knopoff (1960), Slavin and Wolf (1970), Yamada and Sato (1976), and Gupta (1978).

In most of those investigations, the elastic media considered were homogeneous. Not much work has been done on heterogeneous media with corrugated boundary. The reflection and transmission coefficients of elastic waves from flat boundaries for the case of inhomogeneous media with several types of velocity distributions have been studied by Acharya (1970), Saini and Singh (1977b), Singh et al. 
(1978), Malhotra et al. (1982) among others. Chattopadhyay and Pal (1982) studied the propagation of SH-waves in an inhomogeneous medium with irregular interface lying over an initially stressed elastic half space. Gupta (1987) studied the problem of reflection and transmission of SH-waves in laterally and vertically heterogeneous media at an irregular boundary. She used the Rayleigh's method to study the effect of lateral and vertical heterogeneities on the reflection and refraction coefficients, and gave the mathematical treatment to compute these coefficients for first and second order approximation of the corrugation. Zhang et al. (1997) studied seismic waves in a laterally inhomogeneous medium.

Henneke (1972) studied the effect of anisotropy on reflection and refraction of stress wave at a plane boundary between anisotropic media. Daley and Hron (1977) studied reflection and refraction coefficients at a plane interface due to incident $\mathrm{P}$ and SV waves in transversely isotropic elastic media. Saini and Singh (1977a) investigated the effect of transverse isotropy on reflection and transmission coefficients of SH-wave at a plane interface between a homogeneous isotropic half-space and a transversely isotropic halfspace. Rokhlin et al. (1986) studied these coefficients on a plane interface between two generally anisotropic media. The problems of wave propagation from plane boundaries in anisotropic media have also been studied by Musgrave (1960), Keith and Crampin (1977), Mandal (1991), Ruger (1997) among others. Tomar and Saini (1997) studied the reflection and refraction of SH-waves at a corrugated interface between two transversely isotropic elastic half spaces. Recently, Tomar et al. (2002) investigated the reflection and transmission coefficients of SH-waves at corrugated interface between transversely isotropic and visco-elastic solid half-spaces.

The earth medium is complex in nature and full of various types of anisotropy and heterogeneity (see Sheriff and Geldart, 1995; Lay and Wallace, 1995; Shearer, 1999). Moreover, discontinuities in the earth are not perfectly flat (in fact, these discontinuities are stochastically irregular up to some extent). This motivated us to formulate the present model. The present work deals with the problem of reflection and refraction of SH-waves at a corrugated interface between two transversely isotropic and laterally and vertically heterogeneous elastic solid half spaces. Rayleigh's method of approximation has been followed to derive the expressions for reflection and transmission coefficients only for first order approximation of the corrugation due to incident SH-waves. The problems discussed by Tomar and Saini (1997), Gupta (1987) and Asano (1960) have been reduced as particular cases of the present problem.

\section{Formulation of the Problem and Its Solution}

We consider two laterally and vertically heterogeneous anisotropic elastic solid half spaces $H_{1}$ and $H_{2}$, separated by a corrugated interface. The $x$-axis and $y$-axis are on the horizontal plane, whereas the $z$-axis is pointing vertically downwards. The elastic constants, densities and velocities in media $H_{i}$ are given by $M_{i}, N_{i} ; \rho_{i}$ and $\beta_{v_{i}}, \beta_{h_{i}}(i=1,2)$ respectively. The quantities concerning the upper and lower medium will be denoted by subscript 1 and 2, respectively. The equation of the boundary surface (corrugated interface)

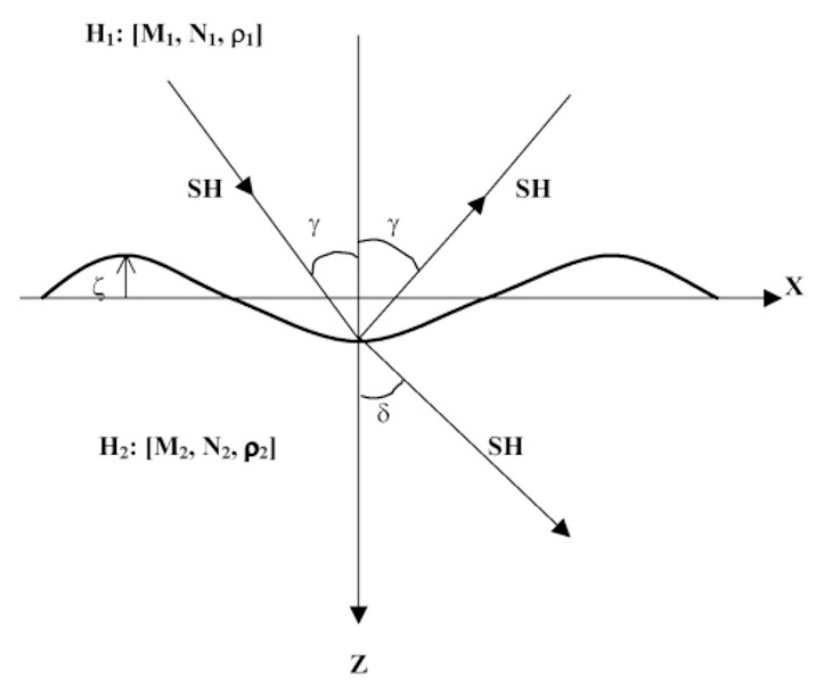

Fig. 1. Geometry of the problem.

is assumed to be

$$
z=\zeta
$$

where $\zeta$ is a periodic function of $x$, independent of $y$, whose mean value is zero. The geometry of the problem is shown in Fig. 1. The Fourier series representation of $\zeta$ is given by

$$
\zeta=\sum_{n=1}^{\infty}\left[\zeta_{n} e^{\imath n k^{*} x}+\zeta_{-n} e^{-\imath n k^{*} x}\right],
$$

where $\zeta_{n}$ and $\zeta_{-n}$ are Fourier expansion coefficients, $n$ is the series expansion order and $\iota=\sqrt{-1}$. Introducing constants $c_{1}, c_{n}$ and $s_{n}$ as

$$
\begin{array}{r}
\zeta_{1}=\zeta_{-1}=\frac{c_{1}}{2}, \quad \zeta_{ \pm n}=\frac{c_{n} \mp \iota s_{n}}{2}, \\
n=2,3,4, \ldots
\end{array}
$$

we obtain

$$
\begin{aligned}
\zeta= & c_{1} \cos k^{*} x+c_{2} \cos 2 k^{*} x+s_{2} \sin 2 k^{*} x+c_{3} \cos 3 k^{*} x \\
& +s_{3} \sin 3 k^{*} x+\cdots+c_{n} \cos n k^{*} x+s_{n} \sin n k^{*} x+\cdots
\end{aligned}
$$

If the interface shape can be expressed by only one cosine term i.e. $\zeta=c_{1} \cos k^{*} x$, the wavelength of corrugation is $2 \pi / k^{*}$, where $k^{*}$ is the wavenumber.

The equation of motion for $\mathrm{SH}$-wave propagation in a transversely isotropic, inhomogeneous elastic medium with zero body forces can be written as follows:

$$
\frac{\partial}{\partial x}\left(N_{i} \frac{\partial V_{i}}{\partial x}\right)+\frac{\partial}{\partial z}\left(M_{i} \frac{\partial V_{i}}{\partial z}\right)=\rho_{i} \frac{\partial^{2} V_{i}}{\partial t^{2}},
$$

where the constants $N_{i}$ and $M_{i}$ and $\rho_{i}$ are defined earlier and $V_{i}$ is $y$-component of the displacement vector in medium $H_{i}$. Since the medium is assumed to be laterally and vertically heterogeneous, we shall take

$$
\left\{N_{i}, M_{i}, \rho_{i}\right\}(x, z)=\left\{N_{i o}, M_{i o}, \rho_{i o}\right\} p(x) r_{i}(z),
$$

where $N_{i 0}, M_{i 0}$ and $\rho_{i 0}$ are constants. We assume the solution of Eq. (4) in the form

$$
V_{i}(x, z, t)=\frac{1}{\sqrt{M_{i}(x, z)}} X(x) Z(z) \exp (\iota \omega t),
$$


where $\omega$ is the angular frequency. Substituting (5) and (6) into (4), we get the following differential equation:

$$
\left[\frac{d^{2} X}{d x^{2}}-\epsilon^{2} X\right] Z=-\frac{M_{i 0}}{N_{i 0}}\left[\frac{d^{2} Z}{d z^{2}}-\left\{b_{i}^{2}-\frac{\rho_{i 0}}{M_{i 0}} \omega^{2}\right\} Z\right]
$$

where $\epsilon$ and $b_{i}$ are given by

$$
\begin{aligned}
\epsilon^{2} & =\frac{1}{2 p} \frac{d^{2} p}{d x^{2}}-\frac{1}{4}\left(\frac{1}{p} \frac{d p}{d x}\right)^{2}, \\
b_{i}^{2} & =\frac{1}{2 r_{i}} \frac{d^{2} r_{i}}{d z^{2}}-\frac{1}{4}\left(\frac{1}{r_{i}} \frac{d r_{i}}{d z}\right)^{2} .
\end{aligned}
$$

For wave propagating in positive $x$-direction, let us take $X(x)=\exp (-\iota k x)$, and plugging it into Eq. (7), we obtain

$$
\frac{d^{2} Z}{d z^{2}}-\frac{N_{i 0}}{M_{i 0}}\left(k^{2}+\epsilon^{2}+\frac{M_{i 0}}{N_{i 0}} b_{i}^{2}-\frac{\omega^{2}}{\beta_{h_{i}}^{2}}\right) Z=0
$$

where $\beta_{h_{i}}=\sqrt{N_{i 0} / \rho_{i 0}}$. The solution of Eq. (9) can be written as

$$
Z=A e^{-s_{i} z}+B e^{s_{i} z},
$$

where $\mathrm{A}$ and $\mathrm{B}$ are constants and

$$
s_{i}=\sqrt{\frac{N_{i 0}}{M_{i 0}}\left(\epsilon^{2}+k^{2}+\frac{M_{i 0}}{N_{i 0}} b^{2}-\frac{\omega^{2}}{\beta_{h_{i}}^{2}}\right)} .
$$

Thus the time harmonic solution of Eq. (4) may be written as

$$
V_{i}=\frac{1}{\sqrt{M_{i 0} p(x) r_{i}(z)}}\left[A e^{-s_{i} z}+B e^{s_{i} z}\right] e^{\iota(\omega t-k x)},
$$

$k=\omega \sin \theta / \beta_{h}$ is the horizontal component of the wave number given by Gupta (1965), $\beta_{h}=\sqrt{N_{0} / \rho_{0}}$ and $\theta$ is the angle between the normal to the wavefront and the positive direction of $z$-axis.

Equations in (8) may be written as

$$
\frac{d}{d p}\left\{\frac{1}{p}\left(\frac{d p}{d x}\right)^{2}\right\}=4 \epsilon^{2}, \quad \frac{d}{d r_{i}}\left\{\frac{1}{r_{i}}\left(\frac{d r_{i}}{d z}\right)^{2}\right\}=4 b_{i}^{2} .
$$

On integrating these equations twice, we obtain

$$
\begin{aligned}
& x+\epsilon_{2}= \pm \frac{1}{2} \int\left(\epsilon^{2} p^{2}+\epsilon_{1} p\right)^{-1 / 2} d p, \\
& z+\epsilon_{4}= \pm \frac{1}{2} \int\left(b_{i}^{2} r_{i}^{2}+\epsilon_{3} r_{i}\right)^{-1 / 2} d r_{i}
\end{aligned}
$$

where $\epsilon_{1}, \epsilon_{2}, \epsilon_{3}$ and $\epsilon_{4}$ are constants of integration. In particular, when $\epsilon_{1}=\epsilon_{2}=0$, we can obtain the form of $p(x)=\exp ( \pm 2 \epsilon x)$ and when $\epsilon_{3}=\epsilon_{4}=0$, we obtain the form of $r_{i}(z)=\exp \left( \pm 2 b_{i} z\right)$. In case, when $\epsilon=0$ and $\epsilon_{1} \epsilon_{2}^{2}=1$, we obtain $p(x)=\left(1+x / \epsilon_{2}\right)^{2}$ and when $b_{i}=0$, $\epsilon_{3} \epsilon_{4}^{2}=1$, we obtain $r_{i}(z)=\left(1+z / \epsilon_{3}\right)^{2}$. If $\epsilon_{1}=\epsilon^{2} \neq 0$, then we obtain $p(x)=\sinh ^{2}\left(x+\epsilon_{2}\right) \epsilon$, and when $\epsilon_{3}=b_{i}^{2} \neq 0$, we obtain $r_{i}(z)=\sinh ^{2}\left(z+\epsilon_{4}\right) b_{i}$ (cf. Singh et al., 1976). Thus we see that the solutions of equations in (8) are of the form $\exp (a x),(1+a x)^{2}$ or $\sinh ^{2} a x$. In the present analysis, we shall take for convenience, the exponential form of $p(x)$ and $r_{i}(x)$.

Considering an incident SH-wave of unit amplitude, the displacement in the medium $H_{1}$ due to both incident and reflected $\mathrm{SH}$-wave, is given by

$$
V^{i n c+r e f l}=\frac{1}{\sqrt{M_{10} p(x) r_{1}(z)}}\left\{e^{-q z}+B e^{q z}\right\} e^{i \omega\left(t-\frac{x \sin \gamma}{\beta_{h_{1}}}\right)},
$$

where $B$ is the amplitude of reflected SH-wave (this reflected wave is called regularly reflected wave), $\gamma$ is the angle of incidence and

$$
q=\sqrt{\frac{N_{10}}{M_{10}}\left(\epsilon^{2}+k^{2}+\frac{M_{10}}{N_{10}} b_{1}^{2}-\frac{\omega^{2}}{\beta_{h_{1}}^{2}}\right)} .
$$

Similarly, the displacement in medium $\mathrm{H}_{2}$ is given by

$$
V^{r e f r}=\frac{1}{\sqrt{M_{20} p(x) r_{2}(z)}} D e^{-u z} e^{i \omega\left(t-\frac{x \sin \delta}{\beta_{h_{2}}}\right)},
$$

where $D$ is the amplitude of refracted SH-wave (this refracted wave is called regularly refracted wave) and

$$
u=\sqrt{\frac{N_{20}}{M_{20}}\left(\epsilon^{2}+k^{2}+\frac{M_{20}}{N_{20}} b_{2}^{2}-\frac{\omega^{2}}{\beta_{h_{2}}^{2}}\right)},
$$

$\delta$ is the angle between the refracted wave and the $z$-axis, connected with the incidence angle $\gamma$ by the Snell's law

$$
\frac{\sin \gamma}{\beta_{h_{1}}}=\frac{\sin \delta}{\beta_{h_{2}}}=\frac{k}{\omega} .
$$

Due to the corrugated interface, the $n$th order spectrum for the scattered reflected waves (these waves are called irregularly reflected waves) is given by (the arguments of $p$ and $r_{i}$ have been dropped)

$$
\begin{aligned}
V^{i r-r e f l}=\frac{1}{\sqrt{M_{10} p r_{1}}} & {\left[B_{n} e^{q_{n} z} e^{\omega \omega\left(t-\frac{x \sin \gamma_{n}}{\beta_{h_{1}}}\right)}\right.} \\
& \left.+B_{n}^{\prime} e^{q_{n}^{\prime} z} e^{\omega\left(t-\frac{x \sin \gamma_{n}^{\prime}}{\beta_{h_{1}}}\right)}\right],
\end{aligned}
$$

where $B_{n}$ and $B_{n}^{\prime}$ are constants and

$$
\begin{aligned}
& q_{n}=\sqrt{\frac{N_{10}}{M_{10}}\left(-\frac{\omega^{2} \cos ^{2} \gamma_{n}}{\beta_{h_{1}}^{2}}+\epsilon^{2}+\frac{M_{10}}{N_{10}} b_{1}^{2}\right)}, \\
& q_{n}^{\prime}=\sqrt{\frac{N_{10}}{M_{10}}\left(-\frac{\omega^{2} \cos ^{2} \gamma_{n}^{\prime}}{\beta_{h_{1}}^{2}}+\epsilon^{2}+\frac{M_{10}}{N_{10}} b_{1}^{2}\right)},
\end{aligned}
$$

$\gamma_{n}$ and $\gamma_{n}^{\prime}$ are the angles of the irregularly reflected waves with the $z$-axis.

Similarly, the $n$th order spectrum for the scattered refracted waves (these waves are called irregularly refracted waves) is given by

$$
\begin{aligned}
& V^{i r-r e f r}=\frac{1}{\sqrt{M_{20} p r_{2}}}\left[D_{n} e^{-u_{n} z} e^{l \omega\left(t-\frac{x \sin \delta_{n}}{\beta_{h_{2}}}\right)}\right. \\
& \left.+D_{n}^{\prime} e^{-u_{n}^{\prime} z} e^{i \omega\left(t-\frac{x \sin \delta_{n}^{\prime}}{\beta_{h_{2}}}\right)}\right]
\end{aligned}
$$


where $D_{n}$ and $D_{n}^{\prime}$ are constants and

$$
\begin{aligned}
& u_{n}=\sqrt{\frac{N_{20}}{M_{20}}\left(-\frac{\omega^{2} \cos ^{2} \delta_{n}}{\beta_{h_{2}}^{2}}+\epsilon^{2}+\frac{M_{20}}{N_{20}} b_{2}^{2}\right)}, \\
& u_{n}^{\prime}=\sqrt{\frac{N_{20}}{M_{20}}\left(-\frac{\omega^{2} \cos ^{2} \delta_{n}^{\prime}}{\beta_{h_{2}}^{2}}+\epsilon^{2}+\frac{M_{20}}{N_{20}} b_{2}^{2}\right)},
\end{aligned}
$$

$\delta_{n}$ and $\delta_{n}^{\prime}$ are the angles of the irregularly refracted waves with the $z$-axis. The angles $\gamma_{n}, \gamma_{n}^{\prime}, \delta_{n}$ and $\delta_{n}^{\prime}$ are given by the following Spectrum theorem (Asano, 1960)

$$
\begin{array}{ll}
\sin \gamma_{n}-\sin \gamma=\frac{n k^{*} \beta_{h_{1}}}{\omega}, & \sin \gamma_{n}^{\prime}-\sin \gamma=-\frac{n k^{*} \beta_{h_{1}}}{\omega}, \\
\sin \delta_{n}-\sin \delta=\frac{n k^{*} \beta_{h_{2}}}{\omega}, & \sin \delta_{n}^{\prime}-\sin \delta=-\frac{n k^{*} \beta_{h_{2}}}{\omega} .
\end{array}
$$

Thus the total displacement $V_{1}$ in the medium $H_{1}$ is the sum of regularly reflected waves, irregularly reflected waves and incident wave:

$$
\begin{aligned}
V_{1}=\frac{1}{\sqrt{M_{10} p r_{1}}}[ & e^{-q z}+B e^{q z}+\sum_{n=1}^{\infty} B_{n} e^{q_{n} z} e^{-\imath n k^{*} x} \\
& \left.+\sum_{n=1}^{\infty} B_{n}^{\prime} e^{q_{n}^{\prime} z} e^{\iota n k^{*} x}\right] e^{\iota \omega\left(t-\frac{x \sin \gamma}{\beta_{h_{1}}}\right)} .
\end{aligned}
$$

Similarly, the total displacement $V_{2}$ in the medium $H_{2}$ is the sum of regularly refracted waves and irregularly refracted waves:

$$
\begin{aligned}
V_{2}=\frac{1}{\sqrt{M_{20} p r_{2}}}\left[D e^{-u z}+\sum_{n=1}^{\infty} D_{n} e^{-u_{n} z} e^{-\imath n k^{*} x}\right. \\
\left.+\sum_{n=1}^{\infty} D_{n}^{\prime} e^{-u_{n}^{\prime} z} e^{\iota n k^{*} x}\right] e^{\iota \omega\left(t-\frac{x \sin \delta}{\beta_{h_{2}}}\right)},
\end{aligned}
$$

The constants $B, D, B_{n}, D_{n}, B_{n}^{\prime}$ and $D_{n}^{\prime}$ can be determined by satisfying the boundary conditions at the interface. In writing Eqs. (25) and (26), we have used Spectrum theorem.

\section{Boundary Conditions}

The boundary conditions to be satisfied at the corrugated interface $z=\zeta$ are the continuity of displacement and traction, that is

(I) $\quad V_{1}=V_{2}$

(II) $M_{1} \frac{\partial V_{1}}{\partial v}=M_{2} \frac{\partial V_{2}}{\partial v}$,

where $v$ denotes the normal to the interface. Following Sokolnikoff (1956) and using relation (5), the boundary condition (II) can be written as

$$
\begin{aligned}
M_{10} p r_{1}(z) & {\left[\frac{\partial V_{1}}{\partial z}-\frac{\partial V_{1}}{\partial x} \zeta^{\prime}\right] \frac{1}{\sqrt{1+\zeta^{\prime 2}}} } \\
= & M_{20} p r_{2}(z)\left[\frac{\partial V_{2}}{\partial z}-\frac{\partial V_{2}}{\partial x} \zeta^{\prime}\right] \frac{1}{\sqrt{1+\zeta^{\prime 2}}},
\end{aligned}
$$

where $\zeta^{\prime}$ is the derivative of $\zeta$ with respect to $x$. Insertion of Eqs. (25) and (26) in (I) and (II), and the use of the following substitutions

$$
\begin{aligned}
& q=\imath Q, \quad u=\imath U, \\
& q_{n}=\imath Q_{n}, \quad u_{n}=\imath U_{n}, \quad q_{n}^{\prime}=\iota Q_{n}^{\prime}, \quad u_{n}^{\prime}=\iota U_{n}^{\prime},
\end{aligned}
$$

where

$$
\begin{aligned}
& Q=\sqrt{\frac{N_{10}}{M_{10}}\left(\frac{\omega^{2}}{\beta_{h_{1}}^{2}}-\epsilon^{2}-k^{2}-\frac{M_{10}}{N_{10}} b_{1}^{2}\right)}, \\
& U=\sqrt{\frac{N_{20}}{M_{20}}\left(\frac{\omega^{2}}{\beta_{h_{2}}^{2}}-\epsilon^{2}-k^{2}-\frac{M_{20}}{N_{20}} b_{2}^{2}\right)}, \\
& Q_{n}=\sqrt{\frac{N_{10}}{M_{10}}\left(\frac{\omega^{2} \cos ^{2} \gamma_{n}}{\beta_{h_{1}}^{2}}-\epsilon^{2}-\frac{M_{10}}{N_{10}} b_{1}^{2}\right)}, \\
& Q_{n}^{\prime}=\sqrt{\frac{N_{10}}{M_{10}}\left(\frac{\omega^{2} \cos ^{2} \gamma_{n}^{\prime}}{\beta_{h_{1}}^{2}}-\epsilon^{2}-\frac{M_{10}}{N_{10}} b_{1}^{2}\right)}, \\
& U_{n}=\sqrt{\frac{N_{20}}{M_{20}}\left(\frac{\omega^{2} \cos ^{2} \delta_{n}}{\beta_{h_{2}}^{2}}-\epsilon^{2}-\frac{M_{20}}{N_{20}} b_{2}^{2}\right)}, \\
& U_{n}^{\prime}=\sqrt{\frac{N_{20}}{M_{20}}\left(\frac{\omega^{2} \cos ^{2} \delta_{n}^{\prime}}{\beta_{h_{2}}^{2}}-\epsilon^{2}-\frac{M_{20}}{N_{20}} b_{2}^{2}\right)},
\end{aligned}
$$

lead to:

$$
\begin{gathered}
\sqrt{r_{2}}\left[e^{-\imath Q \zeta}+B e^{\iota Q \zeta}+\sum_{n=1}^{\infty} B_{n} e^{\iota Q_{n} \zeta} e^{-\imath n k^{*} x}\right. \\
\left.+\sum_{n=1}^{\infty} B_{n}^{\prime} e^{\iota Q_{n}^{\prime} \zeta} e^{\imath n k^{*} x}\right] \\
=\sqrt{m r_{1}}\left[D e^{-\imath U \zeta}+\sum_{n=1}^{\infty} D_{n} e^{-\imath U_{n} \zeta} e^{-\imath n k^{*} x}\right. \\
\left.+\sum_{n=1}^{\infty} D_{n}^{\prime} e^{-\imath U_{n}^{\prime} \zeta} e^{\iota n k^{*} x}\right],
\end{gathered}
$$

and

$$
\begin{aligned}
& \sqrt{m r_{1}}\left[\left\{-Q+\frac{\iota r_{1}^{\prime}}{2 r_{1}}+\left(\frac{\omega \sin \gamma}{\beta_{h_{1}}}-\frac{\iota p^{\prime}}{2 p}\right) \zeta^{\prime}\right\} e^{-\iota Q \zeta}\right. \\
& +B\left\{Q+\frac{\iota r_{1}^{\prime}}{2 r_{1}}+\left(\frac{\omega \sin \gamma}{\beta_{h_{1}}}-\frac{\iota p^{\prime}}{2 p}\right) \zeta^{\prime}\right\} e^{\iota Q \zeta} \\
& +\sum_{n=1}^{\infty} B_{n} e^{-\imath n k^{*} x}\left\{Q_{n}+\frac{\iota r_{1}^{\prime}}{r_{1}}\right. \\
& \left.+\left(\frac{\omega \sin \gamma}{\beta_{h_{1}}}+n k^{*}-\frac{\iota p^{\prime}}{2 p}\right) \zeta^{\prime}\right\} e^{\iota Q_{n} \zeta} \\
& +\sum_{n=1}^{\infty} B_{n}^{\prime} e^{\iota n k^{*} x}\left\{Q_{n}^{\prime}+\frac{\iota r_{1}^{\prime}}{r_{1}}\right. \\
& \left.\left.+\left(\frac{\omega \sin \gamma}{\beta_{h_{1}}}-n k^{*}-\frac{\iota p^{\prime}}{2 p}\right) \zeta^{\prime}\right\} e^{\imath n k^{*} x}\right] \\
& =\sqrt{r_{2}}\left[D\left\{-U+\frac{\iota r_{2}^{\prime}}{2 r_{2}}+\left(\frac{\omega \sin \delta}{\beta_{h_{2}}}-\frac{\iota p^{\prime}}{2 p}\right) \zeta^{\prime}\right\} e^{-\iota U \zeta}\right. \\
& +\sum_{n=1}^{\infty} D_{n} e^{-\imath n k^{*} x}\left\{-U_{n}+\frac{\iota r_{2}^{\prime}}{2 r_{2}}\right. \\
& \left.+\left(\frac{\omega \sin \delta}{\beta_{h_{2}}}+n k^{*}-\frac{\iota p^{\prime}}{2 p}\right) \zeta^{\prime}\right\} e^{-\imath U_{n} \zeta} \\
& +\sum_{n=1}^{\infty} D_{n}^{\prime} e^{\iota n k^{*} x}\left\{-U_{n}^{\prime}+\frac{\iota r_{2}^{\prime}}{r_{2}}\right. \\
& \left.\left.+\left(\frac{\omega \sin \delta}{\beta_{h_{2}}}-n k^{*}-\frac{\iota p^{\prime}}{2 p}\right) \zeta^{\prime}\right\} e^{-\iota U_{n}^{\prime} \zeta}\right] \text {, }
\end{aligned}
$$


where $m=M_{10} / M_{20} ; r_{i}^{\prime}$ and $p^{\prime}$ denote the derivatives of $r_{i}$ and $p$ with respect to $z$ and $x$, respectively. From Eq. (13), with $B=0$ (i.e. the incident wave), Eqs. (15), (26) and (17), the Snell's law; it is apparent that $D, D_{n}$ and $D_{n}^{\prime}$ are connected with the transmission coefficients $T, T_{n}$ and $T_{n}^{\prime}$ through the relation (cf. Singh et al., 1978)

$$
\begin{aligned}
& \left\{T, T_{n}, T_{n}^{\prime}\right\} \\
= & \left\{D e^{-u \zeta}, D_{n} e^{-u_{n} \zeta}, D_{n}^{\prime} e^{-u_{n}^{\prime} \zeta}\right\} e^{q \zeta} \sqrt{\frac{M_{10} r_{1}(\zeta)}{M_{20} r_{2}(\zeta)}} .
\end{aligned}
$$

\section{Solution of the First Order Approximation}

For working out approximate solutions, we assume that the corrugation of the surface $z=\zeta$ is so small that higher powers of $\zeta$ may be neglected, so we have

$$
e^{-\iota Q \zeta}=1-\iota Q \zeta
$$

We shall take

$$
\left\{r_{i}(z), p(x)\right\}=\left\{e^{d_{i} z}, e^{d_{3} x}\right\}, \quad i=1,2 .
$$

where $d_{1}, d_{2}$ and $d_{3}$ are constants. Substituting these into Eqs. (25) and (26), the first order approximation for $B$ and $D$ can be obtained by collecting the terms independent of $x$ and $\zeta$, as follows

$$
\begin{gathered}
(1+B)=\sqrt{m} D \\
\left(-Q+\frac{\iota d_{1}}{2}\right)+B\left(\frac{\iota d_{1}}{2}+Q\right)=\frac{D}{\sqrt{m}}\left(-U+\frac{\iota d_{2}}{2}\right) .
\end{gathered}
$$

These formulae give the values of $B$ and $D$ at the plane interface. $B$ is known as reflection coefficient at the plane interface while $D$ is connected to the transmission coefficient $T$ at the plane interface, by the relation (31). Solving Eqs. (34) and (35), we obtain

$$
\begin{aligned}
& B=\frac{2(-U+m Q)+\iota\left(d_{2}-m d_{1}\right)}{2(U+m Q)-\iota\left(d_{2}-m d_{1}\right)}, \\
& D=\frac{4 \sqrt{m} Q}{2(U+m Q)-\iota\left(d_{2}-m d_{1}\right)} .
\end{aligned}
$$

In order to find the transmission coefficients $T$, we plug the values of $r_{i}$ and $D$ from (33) and (37) into (31), we obtain

$$
T=\frac{4 m Q e^{-(u-q) \zeta} e^{\sqrt{\left(d_{1}-d_{2}\right) \zeta}}}{2(U+m Q)-\iota\left(d_{2}-m d_{1}\right)} .
$$

Approximating the exponential terms $e^{-(u-q) \zeta}$ and $e^{\sqrt{\left(d_{1}-d_{2}\right) \zeta}}$, similar to (32) and collecting the constant term (i.e. independent of $x$ and $\zeta$ ), the transmission coefficient $T$ at the plane interface is obtained as

$$
T=\frac{4 m Q}{2(U+m Q)-\iota\left(d_{2}-m d_{1}\right)} .
$$

Keeping in view the geometry of the problem, we see that expressions of $B$ and $D$ in (36) and (37) are same as obtained by Singh et al. (1978) for the relevant problem.
Next, for the solution of first order approximation for $B_{n}$ and $D_{n}$, we collect the coefficients of $e^{-\imath n k^{*} x}$ and obtaining

$$
\begin{gathered}
B_{n}-\sqrt{m} D_{n}=\left[\sqrt{m}\left(\frac{d_{1}}{2}-\iota U\right) D\right. \\
\left.-\frac{d_{2}}{2}(1+B)+\iota Q(1-B)\right] \zeta_{-n} \\
\left(\iota Q_{n}-\frac{d_{1}}{2}\right) B_{n}+\frac{1}{\sqrt{m}}\left(\frac{d_{2}}{2}+\iota U_{n}\right) D_{n} \\
=\left[\left\{Q^{2}+\frac{d_{1}^{2}}{4}+\iota n k^{*}\left(\frac{d_{3}}{2}+\frac{\iota \omega \sin \gamma}{\beta_{h_{1}}}\right)\right\} \times(1+B)\right. \\
\left.\quad-\frac{1}{\sqrt{m}}\left\{U^{2}+\frac{d_{2}^{2}}{4}+\iota n k^{*}\left(\frac{d_{3}}{2}+\frac{\iota \omega \sin \delta}{\beta_{h_{2}}}\right)\right\} D\right] \zeta_{-n} .
\end{gathered}
$$

Similarly, equating the coefficients of $e^{i n k^{*} x}$, we obtain the first order approximation for $B_{n}^{\prime}$ and $D_{n}^{\prime}$ as follows

$$
\begin{aligned}
B_{n}^{\prime}-\sqrt{m} D_{n}^{\prime}=[ & \sqrt{m}\left(\frac{d_{1}}{2}-\iota U\right) D \\
& \left.+\iota Q(1-B)-\frac{d_{2}}{2}(1+B)\right] \zeta_{n},
\end{aligned}
$$

$$
\begin{aligned}
B_{n}^{\prime}\left(\iota Q_{n}^{\prime}-\frac{d_{1}}{2}\right)+\frac{1}{\sqrt{m}}\left(\frac{d_{2}}{2}+\iota U_{n}^{\prime}\right) D_{n}^{\prime} \\
=\left[\left\{Q^{2}+\frac{d_{1}^{2}}{4}-\iota n k^{*}\left(\frac{d_{3}}{2}+\frac{\iota \omega \sin \gamma}{\beta_{h_{1}}}\right) \times(1+B)\right.\right. \\
\left.\quad-\frac{1}{\sqrt{m}}\left\{U^{2}+\frac{d_{2}^{2}}{4}-\iota n k^{*}\left(\frac{d_{3}}{2}+\frac{\iota \omega \sin \delta}{\beta_{h_{2}}}\right)\right\} D\right] \zeta_{n} .
\end{aligned}
$$

The Eqs. (39)-(42) give the values of $B_{n}, D_{n}, B_{n}^{\prime}$ and $D_{n}^{\prime}$ as follows

$$
\begin{array}{rlrl}
B_{n} & =\frac{\Delta_{B_{n}}}{\Delta_{n}}, & D_{n}=\frac{\Delta_{D_{n}}}{\Delta_{n}}, \\
B_{n}^{\prime}=\frac{\Delta_{B_{n}^{\prime}}}{\Delta_{n}^{\prime}}, & D_{n}^{\prime}=\frac{\Delta_{D_{n}^{\prime}}}{\Delta_{n}^{\prime}},
\end{array}
$$

where

$$
\begin{aligned}
\Delta_{B_{n}}=\iota \zeta_{-n}\left[(1+B)\left\{m\left(\frac{n k^{*} \omega \sin \gamma}{\beta_{h_{1}}}-\frac{d_{1}^{2}}{4}-Q^{2}\right)+\frac{d_{2}^{2}}{4}\right\}\right. \\
+(1-B) Q U_{n}+D \sqrt{m} \\
\cdot\left(-U U_{n}-\frac{d_{1} d_{2}}{4}-\frac{n k^{*} \omega \sin \gamma}{\beta_{h_{1}}}\right. \\
\left.+\frac{d_{2}^{2}}{4}+U^{2}\right) \\
+\left\{(1+B)\left(\frac{U_{n} d_{2}}{2}-\frac{m d_{3} n k^{*}}{2}\right)\right. \\
\left.\left.\left.\quad-(1-B) \frac{Q d_{2}}{2}{ }^{2}\right)\right\}\right] \\
\left.\left.+D \sqrt{m}\left(\frac{U d_{2}}{2}-\frac{U_{n} d_{1}}{2}+\frac{n k^{*} d_{3}}{2}\right)\right\}\right]
\end{aligned}
$$




$$
\begin{aligned}
\Delta_{D_{n}}=\iota \zeta_{-n}[ & (1+B) \sqrt{m}\left(\frac{n k^{*} \omega \sin \gamma}{\beta_{h_{1}}}-\frac{d_{1}^{2}}{4}\right. \\
- & \left.Q^{2}+\frac{d_{1} d_{2}}{4}\right) \\
& -\sqrt{m}(1-B) Q Q_{n} \\
+ & D\left(U^{2}+\frac{d_{2}^{2}}{4}\right. \\
& \left.-\frac{n k^{*} \omega \sin \gamma}{\beta_{h_{1}}}-\frac{m d_{1}^{2}}{4}+m U Q_{n}\right) \\
+ & \left\{(1+B) \sqrt{m}\left(-\frac{Q_{n} d_{2}}{2}-\frac{d_{3} n k^{*}}{2}\right)\right. \\
& -\sqrt{m}(1-B) \frac{Q d_{1}}{2} \\
& \left.\left.+D\left(\frac{n k^{*} d_{3}}{2}+\frac{m U d_{1}}{2}+\frac{m Q_{n} d_{1}}{2}\right)\right\}\right],
\end{aligned}
$$

$$
\begin{aligned}
\Delta_{B_{n}^{\prime}}=\iota \zeta_{n}[ & (1+B)\left\{-m\left(\frac{n k^{*} \omega \sin \gamma}{\beta_{h_{1}}}\right.\right. \\
& \left.\left.+\frac{d_{1}^{2}}{4}+Q^{2}\right)+\frac{d_{2}^{2}}{4}\right\} \\
& +(1-B) Q U_{n}^{\prime} \\
& -\sqrt{m} D\left(U U_{n}^{\prime}+\frac{d_{1} d_{2}}{4}\right. \\
+ & \left.-\frac{n k^{*} \omega \sin \gamma}{\beta_{h_{1}}}-\frac{d_{2}^{2}}{4}-U^{2}\right) \\
& -(1-B)\left(\frac{U_{n}^{\prime} d_{2}}{2}+\frac{m d_{3} n k^{*}}{2}\right) \\
& \left.\left.-\sqrt{m} D\left(\frac{U_{n}}{2}-\frac{U d_{2}}{2}+\frac{n k^{*} d_{3}}{2}\right)\right\}\right],
\end{aligned}
$$

$$
\begin{aligned}
\Delta_{D_{n}^{\prime}}=\imath \zeta_{n}[ & (1+B) \sqrt{m}\left(\frac{n k^{*} \omega \sin \gamma}{\beta_{h_{1}}}\right. \\
& \left.+\frac{d_{1}^{2}}{4}+Q^{2}-\frac{d_{1} d_{2}}{4}\right) \\
& -\sqrt{m}(1-B) Q Q_{n}^{\prime} \\
+ & D\left(U^{2}+\frac{d_{2}^{2}}{4}+\frac{n k^{*} \omega \sin \gamma}{\beta_{h_{1}}}\right. \\
+ & \quad\left\{\begin{array}{l}
m d_{1}^{2} \\
4
\end{array}+m U Q_{n}^{\prime}\right) \\
& -\sqrt{m}(1-B) \frac{Q d_{1}}{2}\left(\frac{d_{3} n k^{*}}{2}-\frac{Q_{n}^{\prime} d_{2}}{2}\right) \\
& \left.\left.-D\left(\frac{n k^{*} d_{3}}{2}-\frac{m U d_{1}}{2}-\frac{m Q_{n}^{\prime} d_{1}}{2}\right)\right\}\right],
\end{aligned}
$$

$$
\begin{aligned}
& \Delta_{n}=\left(U_{n}+m Q_{n}\right)+\frac{\iota}{2}\left(d_{1} m-d_{2}\right), \\
& \Delta_{n}^{\prime}=\left(U_{n}^{\prime}+m Q_{n}^{\prime}\right)+\frac{\iota}{2}\left(d_{1} m-d_{2}\right) .
\end{aligned}
$$

The values of $B$ and $D$ appearing in the above expressions are given by (36) and (37). Here $B_{n}$ and $B_{n}^{\prime}$ are reflection coefficients for the first order approximation of the corrugation, while the transmission coefficients for the first order approximation of the corrugation, the $T_{n}$ and $T_{n}^{\prime}$ can be obtained from relation (31), on the similar way as $T$ was obtained earlier, as

$$
T_{n}=\sqrt{m} D_{n}, \quad T_{n}^{\prime}=\sqrt{m} D_{n}^{\prime} .
$$

\subsection{Special case}

If we consider $\zeta_{n}=\zeta_{-n}=0,(n \neq 1) ; \zeta_{1}=\zeta_{-1}=c / 2$ then the boundary surface is given by $z=c \cos k^{*} x$, where $c$ is the amplitude of the corrugation. From Eq. (43), we obtain the following formulae of $B_{1}, D_{1}, B_{1}^{\prime}$ and $D_{1}^{\prime}$ for the first order approximation of the corrugation as

$$
\begin{array}{rlrl}
B_{1} & =\frac{\Delta_{B_{1}}}{\Delta_{1}}, & D_{1}=\frac{\Delta_{D_{1}}}{\Delta_{1}}, \\
B_{1}^{\prime}=\frac{\Delta_{B_{1}^{\prime}}}{\Delta_{1}^{\prime}}, & D_{1}^{\prime}=\frac{\Delta_{D_{1}^{\prime}}}{\Delta_{1}^{\prime}},
\end{array}
$$

where the values of $\Delta_{B_{1}}, \Delta_{D_{1}}, \Delta_{B_{1}^{\prime}}, \Delta_{D_{1}^{\prime}}, \Delta_{1}$ and $\Delta_{1}^{\prime}$ are given in Appendix A. The values of the transmission coefficients $T_{1}$ and $T_{1}^{\prime}$, in the present case, are given by

$$
T_{1}=\sqrt{m} D_{1}, \quad T_{1}^{\prime}=\sqrt{m} D_{1}^{\prime} .
$$

\subsection{Particular cases}

(a) When lateral and vertical heterogeneity of the media is removed then the medium $H_{1}$ and $H_{2}$ becomes transversely isotropic. Thus in this case, plugging $p(x)=r_{1}(x)=$ $r_{2}(x)=1$ into Eq. (8), we obtain $\epsilon=b_{1}=b_{2}=0$ and hence the values of $Q, U, Q_{n}, U_{n}, Q_{n}^{\prime}$ and $U_{n}^{\prime}$ given in Eq. (28) through the Eqs. (14), (16), (19), (20), (22) and (23) reduce to

$$
\begin{aligned}
& Q=\frac{\omega}{\beta_{h_{1}}} \sqrt{\frac{N_{10}}{M_{10}}} \cos \gamma, \\
& U=\frac{\omega}{\beta_{h_{1}}} \sqrt{\frac{N_{20}}{M_{20}}\left(\frac{\beta_{h_{1}}^{2}}{\beta_{h_{2}}^{2}}-\sin ^{2} \gamma\right)}=R, \\
& Q_{n}=\frac{\omega}{\beta_{h_{1}}} \sqrt{\frac{N_{10}}{M_{10}}} \cos \gamma_{n}, \\
& U_{n}=\frac{\omega}{\beta_{h_{1}}} \sqrt{\frac{N_{20}}{M_{20}}\left(\frac{\beta_{h_{1}}^{2}}{\beta_{h_{2}}^{2}}-\sin ^{2} \gamma_{n}\right)}=R_{n}, \\
& Q_{n}^{\prime}=\frac{\omega}{\beta_{h_{1}}} \sqrt{\frac{N_{10}}{M_{10}}} \cos \gamma_{n}^{\prime}, \\
& U_{n}^{\prime}=\frac{\omega}{\beta_{h_{1}}} \sqrt{\frac{N_{20}}{M_{20}}\left(\frac{\beta_{h_{1}}^{2}}{\beta_{h_{2}}^{2}}-\sin ^{2} \gamma_{n}^{\prime}\right)}=R_{n}^{\prime},
\end{aligned}
$$




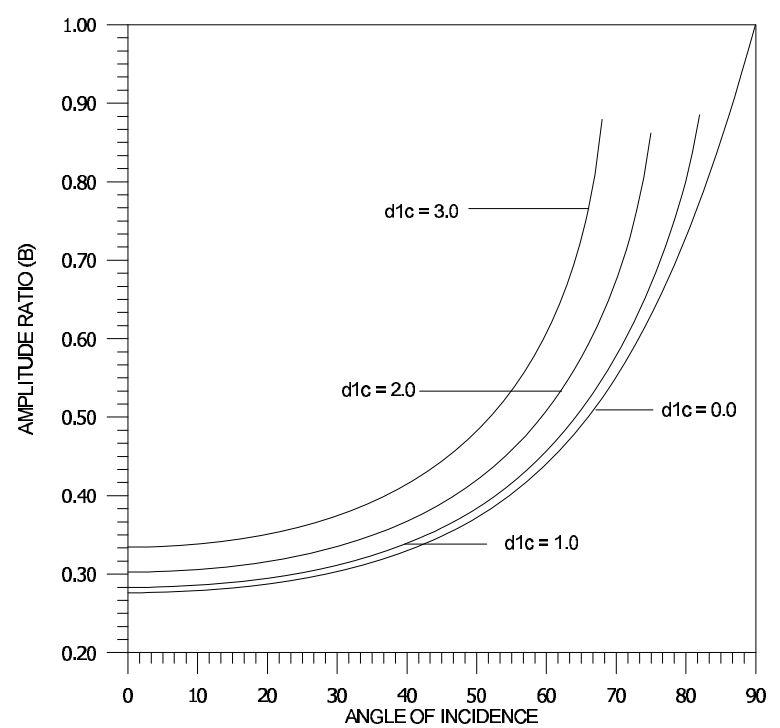

Fig. 2. Variation of the Reflection coefficient at the plane interface (B) with the angle of incidence $\gamma$ : when $d_{2} c=0=d_{3} c$ and $d_{1} c=0.0,1.0,2.0,3.0$.

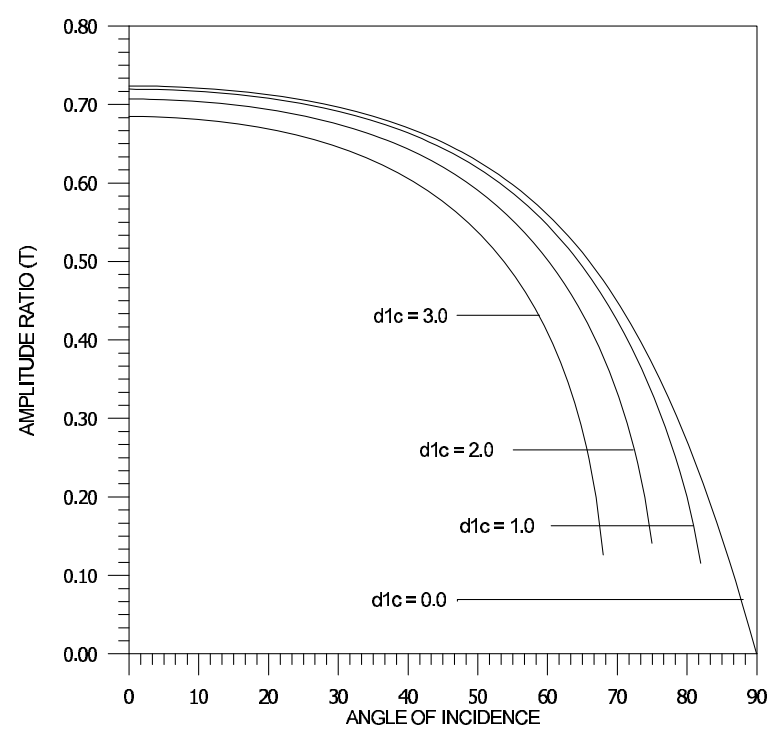

Fig. 3. Variation of the Transmission coefficient at the plane interface (T) with the angle of incidence $\gamma$ : when $d_{2} c=0=d_{3} c$ and $d_{1} c=0.0,1.0,2.0,3.0$.

With the help of these values, the coefficients given by Eqs. (36) and (37) for the plane interface, become

$$
B=\frac{\frac{M_{10}}{M_{20}} \sqrt{\frac{N_{10}}{M_{10}}} \cos \gamma-\sqrt{\frac{N_{20}}{M_{20}}\left(\frac{\beta_{h_{1}}^{2}}{\beta_{h_{2}}^{2}}-\sin ^{2} \gamma\right)}}{\frac{M_{10}}{M_{20}} \sqrt{\frac{N_{10}}{M_{10}}} \cos \gamma+\sqrt{\frac{N_{20}}{M_{20}}\left(\frac{\beta_{h_{1}}^{2}}{\beta_{h_{2}}^{2}}-\sin ^{2} \gamma\right)}}
$$

and

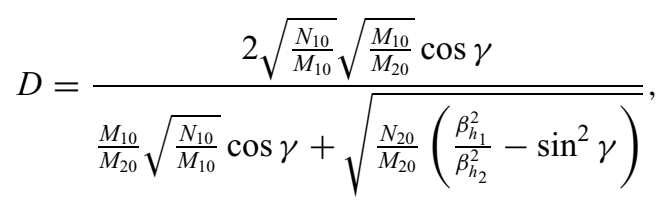

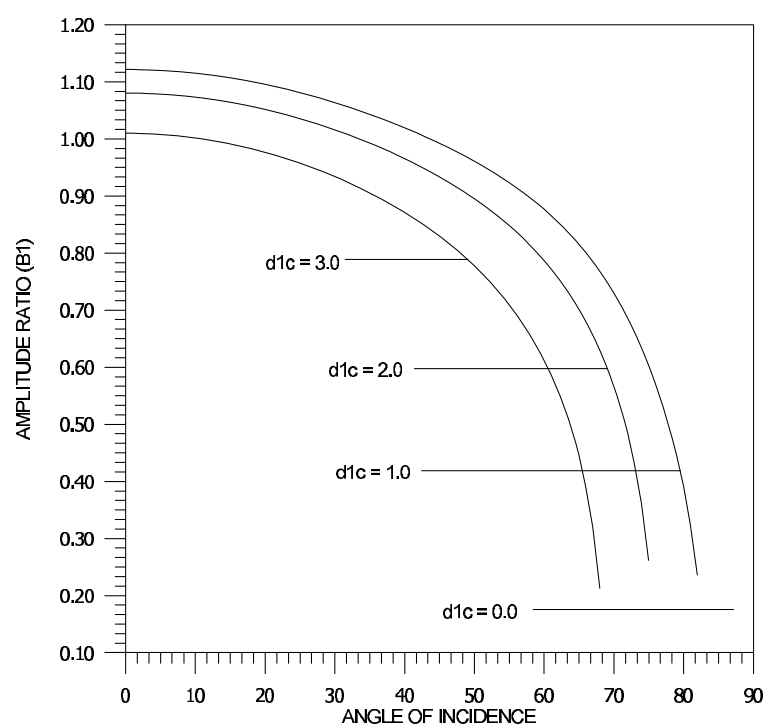

Fig. 4. Variation of the Reflection coefficient for the first order approximation of corrugation $\left(B_{1}\right)$ with the angle of incidence $\gamma$ : when $d_{2} c=0=d_{3} c$ and $d_{1} c=0.0,1.0,2.0,3.0$.

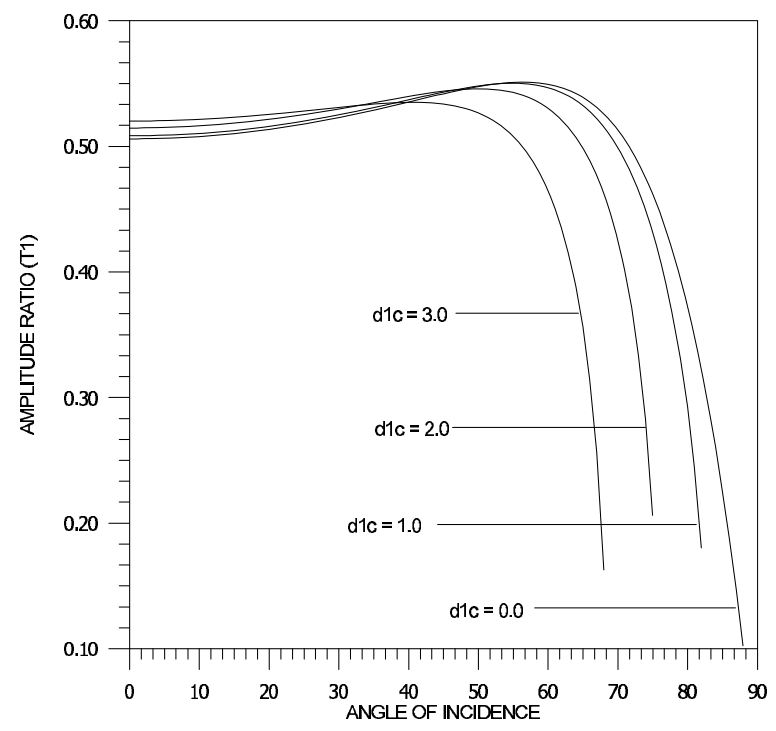

Fig. 5. Variation of the Transmission coefficient for the first order approximation of corrugation $\left(T_{1}\right)$ with the angle of incidence $\gamma$ : when $d_{2} c=0=d_{3} c$ and $d_{1} c=0.0,1.0,2.0,3.0$.

and $B_{1}, D_{1}, B_{1}^{\prime}$ and $D_{1}^{\prime}$ are given in Appendix B. One can verify that using the relations, explained earlier

$$
\left\{T, T_{1}, T_{1}^{\prime}\right\}=\sqrt{m}\left\{D, D_{1}, D_{1}^{\prime}\right\}
$$

the reflection and transmission coefficients obtained in this case, coincide with those of Tomar and Saini (1997).

(b) When transverse isotropy of the media is removed, we shall be left with heterogeneity property in both the media. In this case, putting $N_{10}=M_{10}$ and $N_{20}=M_{20}$, in Eq. (28) 


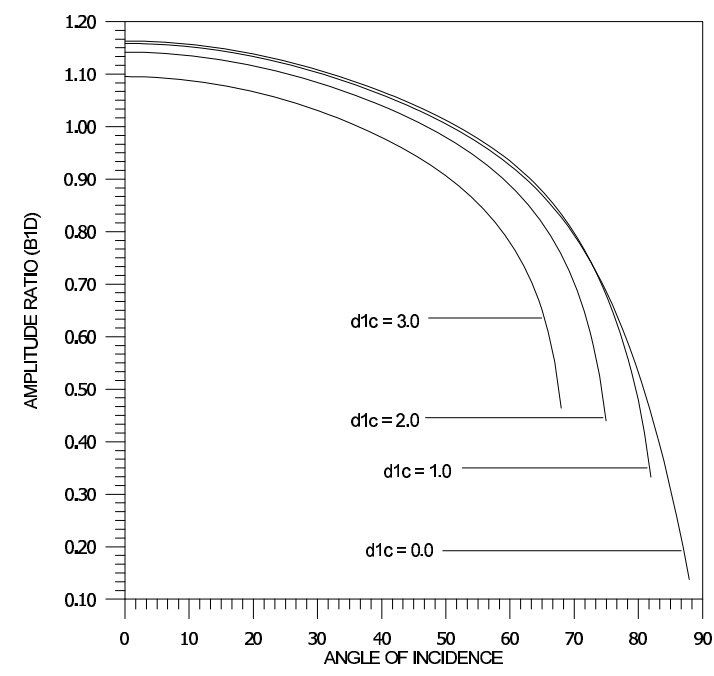

Fig. 6. Variation of the Reflection coefficient for the first order approximation of corrugation $\left(B_{1}^{\prime}\right)$ with the angle of incidence $\gamma$ : when $d_{2} c=0=d_{3} c$ and $d_{1} c=0.0,1.0,2.0,3.0$.

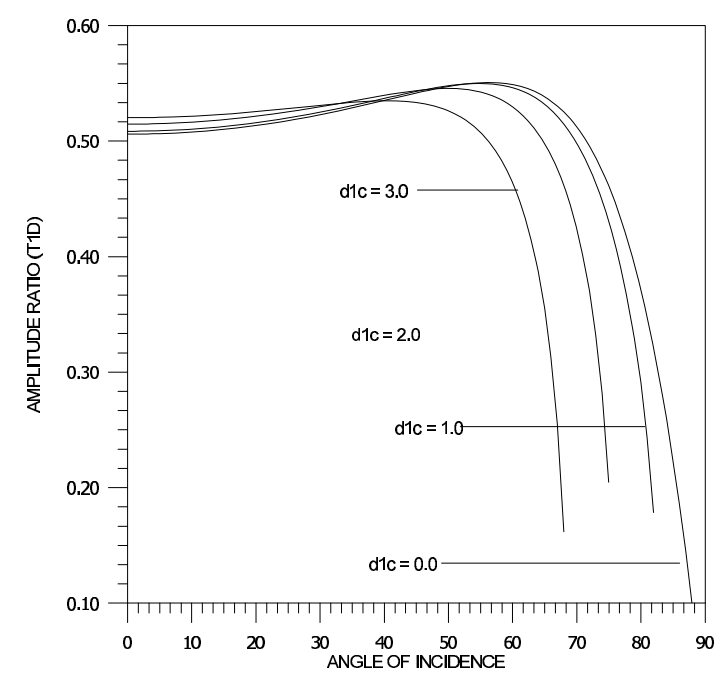

Fig. 7. Variation of the Transmission coefficient for the first order approximation of corrugation $\left(T_{1}^{\prime}\right)$ with the angle of incidence $\gamma$ : when $d_{2} c=0=d_{3} c$ and $d_{1} c=0.0,1.0,2.0,3.0$.

with the Eqs. (14), (16), (19), (20), (22) and (23), we have

$$
\begin{gathered}
Q=-\imath\left(\epsilon^{2}+b_{1}^{2}-\frac{\omega^{2} \cos ^{2} \gamma}{\beta_{h_{1}}^{2}}\right)^{1 / 2}, \\
U=-\imath\left(\epsilon^{2}+b_{2}^{2}-\frac{\omega^{2} \cos ^{2} \delta}{\beta_{h_{2}}^{2}}\right)^{1 / 2}, \\
Q_{n}=-\imath\left(\epsilon^{2}+b_{1}^{2}-\frac{\omega^{2} \cos ^{2} \gamma_{n}}{\beta_{h_{1}}^{2}}\right)^{1 / 2}, \\
U_{n}=-\iota\left(\epsilon^{2}+b_{2}^{2}-\frac{\omega^{2}}{\beta_{h_{2}}^{2}} \cos ^{2} \delta_{n}\right)^{1 / 2}, \\
Q_{n}^{\prime}=-\iota\left(\epsilon^{2}+b_{1}^{2}-\frac{\omega^{2} \cos ^{2} \gamma_{n}^{\prime}}{\beta_{h_{1}}^{2}}\right)^{1 / 2}, \\
U_{n}^{\prime}=-\iota\left(\epsilon^{2}+b_{2}^{2}-\frac{\omega^{2} \cos ^{2} \delta_{n}^{\prime}}{\beta_{h_{2}}^{2}}\right)^{1 / 2} .
\end{gathered}
$$

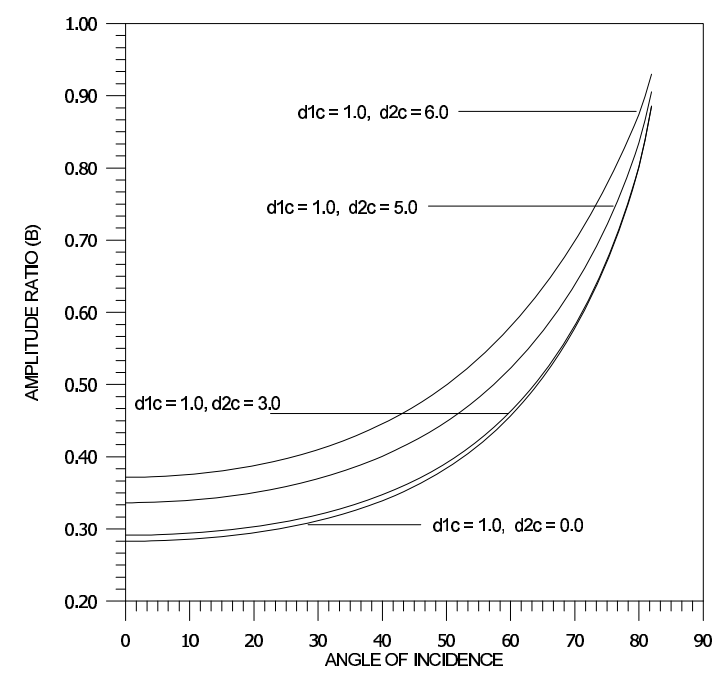

Fig. 8. Variations of the Reflection coefficient at the plane interface (B) with $\gamma$ : when $d_{3} c=0.0, d_{1} c=1.0$ and $d_{2} c=0.0,3.0,5.0,6.0$.

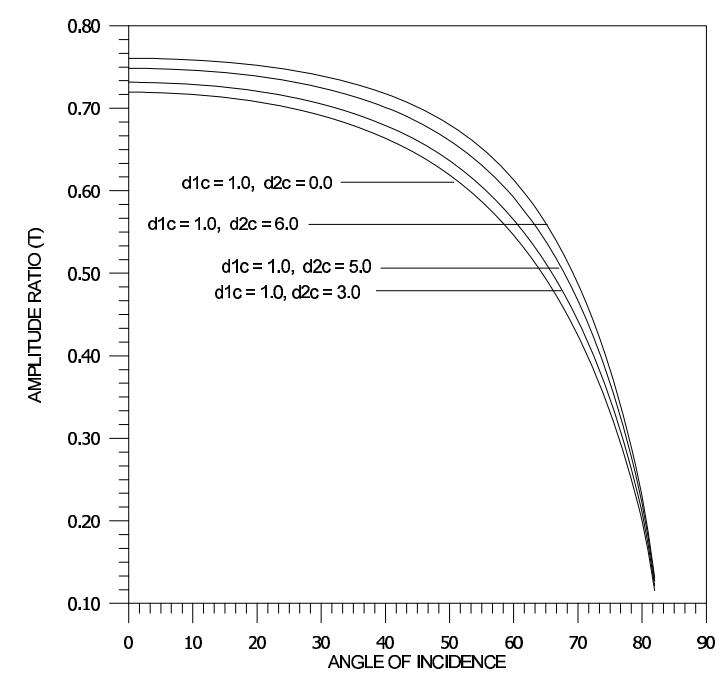

Fig. 9. Variations of the Transmission coefficient at the plane interface (T) with $\gamma$ : when $d_{3} c=0.0, d_{1} c=1.0$ and $d_{2} c=0.0,3.0,5.0,6.0$.

Using these modified values, the reflection and transmission coefficients for first order approximation can be obtained with the help of Eq. (43) and making changes accordingly in the Appendix-I. Also, it can be verified that in the present case, the boundary conditions (39)-(42) matches with those of Gupta (1987) for the corresponding problem.

(c) When only lateral heterogeneity of both media is removed, we are left with vertically heterogeneous and transversely isotropic media. In this case, $p(x)=1$ so that $\epsilon=0$. Putting these values in the Eq. (28) with Eqs. (14), (16), (19), (20), (22), (23), we get

$$
\begin{array}{r}
Q=-\iota \sqrt{\frac{N_{10}}{M_{10}}\left(\frac{M_{10}}{N_{10}} b_{1}^{2}-\frac{\omega^{2}}{\beta_{h_{1}}^{2}} \cos ^{2} \gamma\right)}, \\
U=\iota \sqrt{\frac{N_{20}}{M_{20}}\left(\frac{M_{20}}{N_{20}} b_{2}^{2}-\frac{\omega^{2}}{\beta_{h_{2}}^{2}} \cos ^{2} \delta\right)},
\end{array}
$$




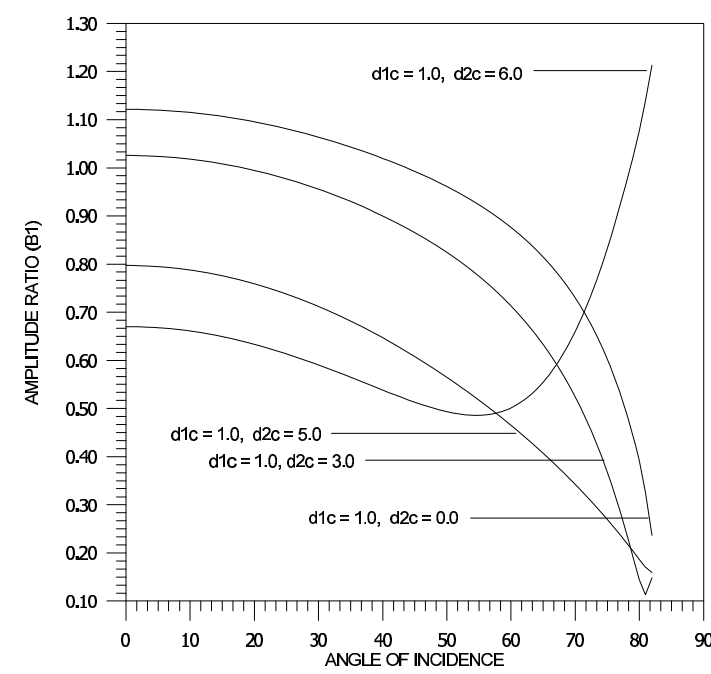

Fig. 10. Variations of the Reflection coefficient for the first order approximation of the corrugation $\left(B_{1}\right)$ with $\gamma$ : when $d_{3} c=0.0, d_{1} c=1.0$ and $d_{2} c=0.0,3.0,5.0,6.0$.

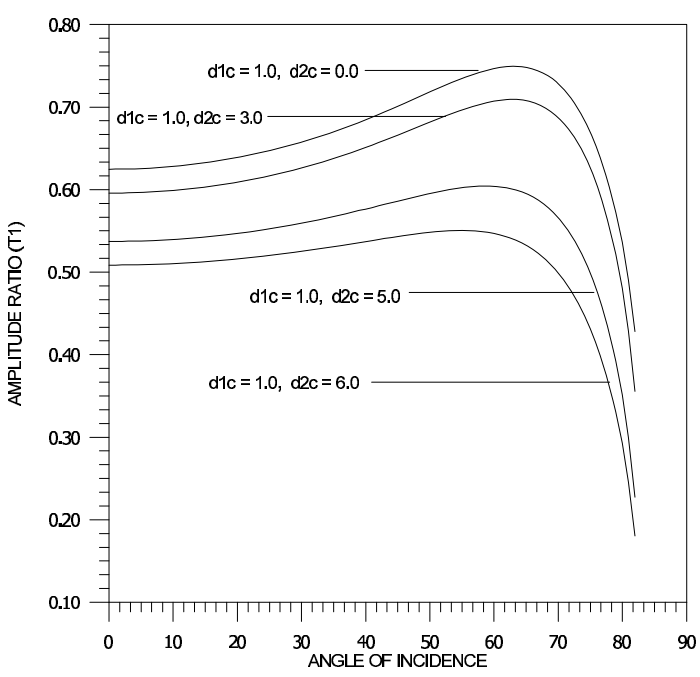

Fig. 11. Variations of the Transmission coefficient for the first order approximation of the corrugation $\left(T_{1}\right)$ with $\gamma$ : when $d_{3} c=0.0, d_{1} c=1.0$ and $d_{2} c=0.0,3.0,5.0,6.0$.

$$
\begin{aligned}
& Q_{n}=-\iota \sqrt{\frac{N_{10}}{M_{10}}\left(\frac{M_{10}}{N_{10}} b_{1}^{2}-\frac{\omega^{2}}{\beta_{h_{1}}^{2}} \cos ^{2} \gamma_{n}\right)}, \\
& U_{n}=\iota \sqrt{\frac{N_{20}}{M_{20}}\left(\frac{M_{20}}{N_{20}} b_{2}^{2}-\frac{\omega^{2}}{\beta_{h_{2}}^{2}} \cos ^{2} \delta_{n}\right)}, \\
& Q_{n}^{\prime}=-\iota \sqrt{\frac{N_{10}}{M_{10}}\left(\frac{M_{10}}{N_{10}} b_{1}^{2}-\frac{\omega^{2}}{\beta_{h_{1}}^{2}} \cos ^{2} \gamma_{n}^{\prime}\right),} \\
& U_{n}^{\prime}=\iota \sqrt{\frac{N_{20}}{M_{20}}\left(\frac{M_{20}}{N_{20}} b_{2}^{2}-\frac{\omega^{2}}{\beta_{h_{2}}^{2}} \cos ^{2} \delta_{n}^{\prime}\right) .}
\end{aligned}
$$

With these modified values, the reflection and transmission coefficients for first order approximation are given by Eq. (43) with (44).

(d) When vertical heterogeneity is removed, we shall have laterally heterogeneous and transversely isotropic media. In

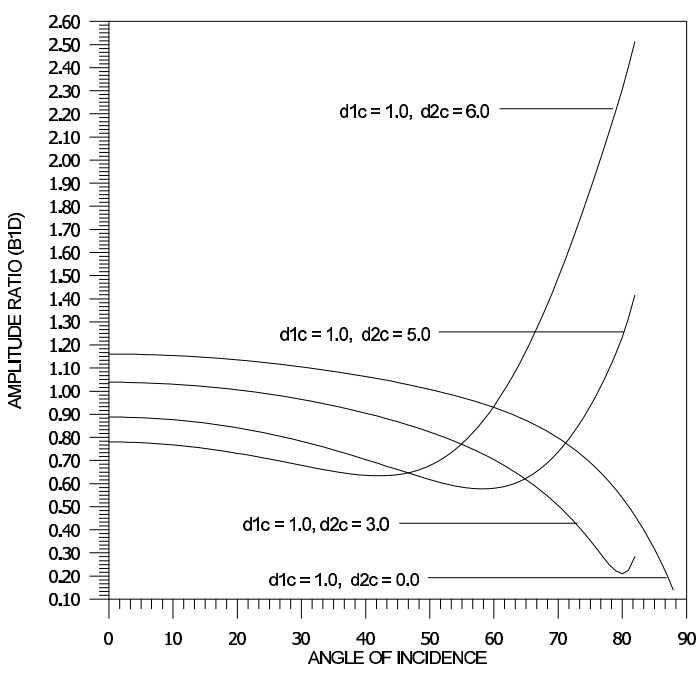

Fig. 12. Variations of the Reflection coefficient for the first order approximation of the corrugation $\left(B_{1}^{\prime}\right)$ with $\gamma$ : when $d_{3} c=0.0, d_{1} c=1.0$ and $d_{2} c=0.0,3.0,5.0,6.0$.

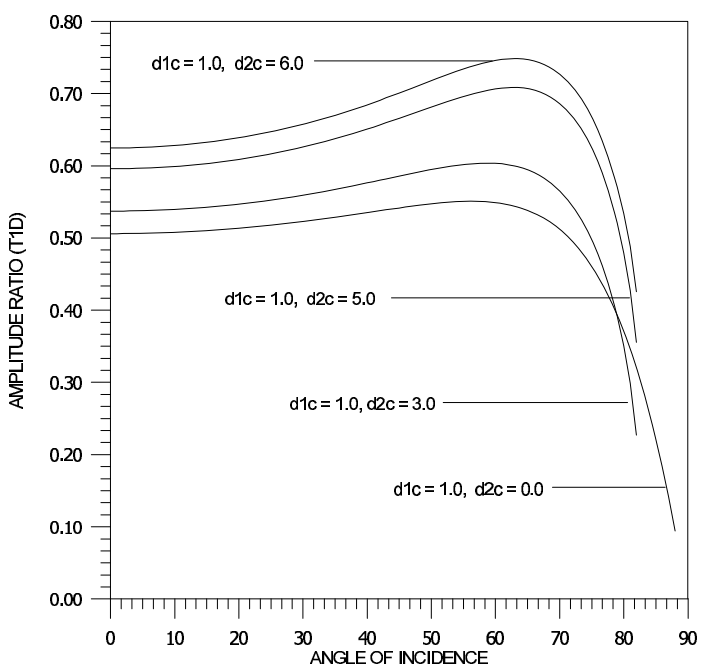

Fig. 13. Variations of the Transmission coefficient for the first order approximation of the corrugation $\left(T_{1}^{\prime}\right)$ with $\gamma$ : when $d_{3} c=0.0, d_{1} c=1.0$ and $d_{2} c=0.0,3.0,5.0,6.0$.

that case, $r_{1}(z)=r_{2}(z)=1$ so that $b_{1}=b_{2}=0$ and hence the values of $Q, U, Q_{n}, U_{n}, Q_{n}^{\prime}$ and $U_{n}^{\prime}$ given in Eq. (28) reduce to

$$
\begin{gathered}
Q=\iota \sqrt{\frac{N_{10}}{M_{10}}\left(\epsilon^{2}-\frac{\omega^{2}}{\beta_{h_{1}}^{2}} \cos ^{2} \gamma\right),} \\
U=\iota \sqrt{\frac{N_{10}}{M_{10}}\left(\epsilon^{2}-\frac{\omega^{2}}{\beta_{h_{2}}^{2}} \cos ^{2} \delta\right),} \\
Q_{n}=\iota \sqrt{\frac{N_{10}}{M_{10}}\left(\epsilon^{2}-\frac{\omega^{2}}{\beta_{h_{1}}^{2}} \cos ^{2} \gamma_{n}\right),} \\
U_{n}=\iota \sqrt{\frac{N_{10}}{M_{10}}\left(\epsilon^{2}-\frac{\omega^{2}}{\beta_{h_{2}}^{2}} \cos ^{2} \delta_{n}\right)},
\end{gathered}
$$




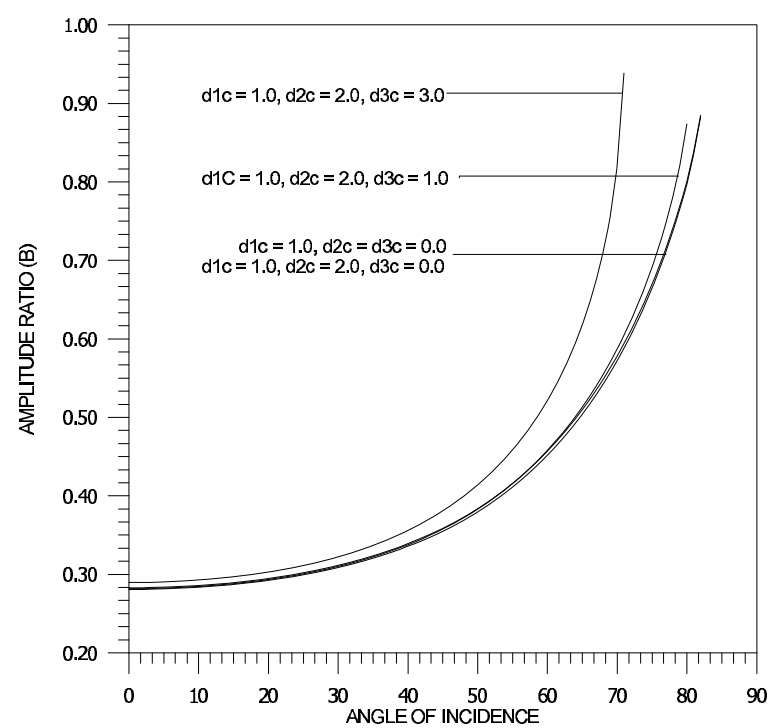

Fig. 14. Variations of the Reflection coefficient at the plane interface (B) with $\gamma$ : when $\left(d_{1} c, d_{2} c, d_{3} c\right)=\left(\begin{array}{lll}1.0 & 0.0,0.0\end{array}\right)$; $(1.0,2.0,0.0) ;(1.0,2.0,1.0) ;(1.0,2.0,3.0)$.

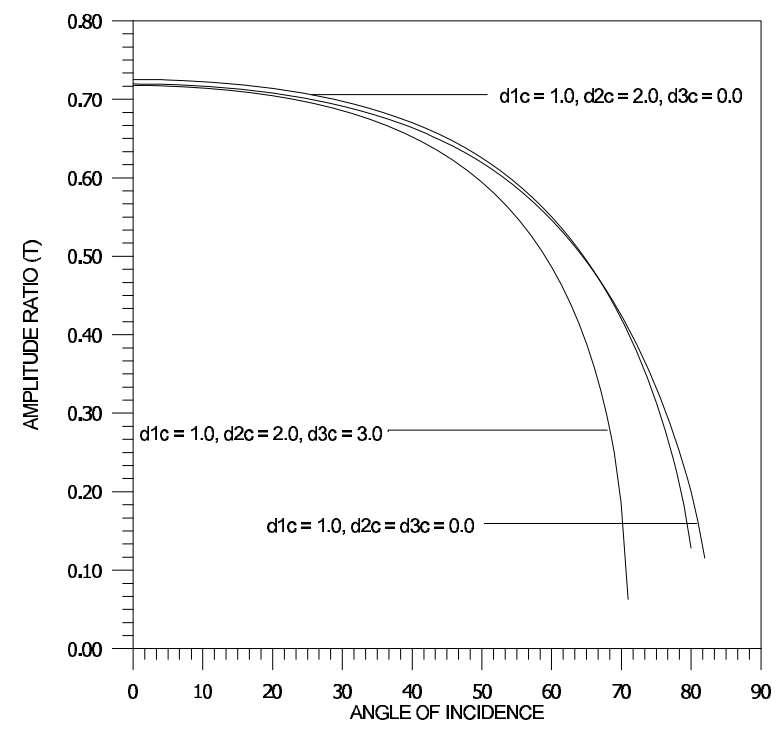

Fig. 15. Variations of the Transmission coefficient at the plane interface (T) with $\gamma$ : when $\left(d_{1} c, d_{2} c, d_{3} c\right)=\left(\begin{array}{lll}1.0, & 0.0,0.0\end{array}\right)$; $(1.0,2.0,0.0) ;(1.0,2.0,1.0) ;(1.0,2.0,3.0)$.

$$
\begin{aligned}
Q_{n}^{\prime}=\iota \sqrt{\frac{N_{10}}{M_{10}}\left(\epsilon^{2}-\frac{\omega^{2}}{\beta_{h_{1}}^{2}} \cos ^{2} \gamma_{n}^{\prime}\right)}, \\
U_{n}^{\prime}=\iota \sqrt{\frac{N_{10}}{M_{10}}\left(\epsilon^{2}-\frac{\omega^{2}}{\beta_{h_{2}}^{2}} \cos ^{2} \delta_{n}^{\prime}\right)} .
\end{aligned}
$$

With these modified values, the reflection and transmission coefficients for first order approximation are given by the Eqs. (43) with (44).

(e)When both the heterogeneity and the transverse isotropy of the medium are removed, the medium $H_{1}$ and $H_{2}$ becomes homogeneous and isotropic elastic half spaces. In this case, by plugging $p(x)=r_{1}(x)=r_{2}(x)=1, N_{10}=$ $M_{10}$ and $N_{20}=M_{20}$ into Eq. (8), we get $\epsilon=0$ and $b_{1}=$ $b_{2}=0$. And the values of $Q, U, Q_{n}, U_{n}, Q_{n}^{\prime}$ and $U_{n}^{\prime}$,

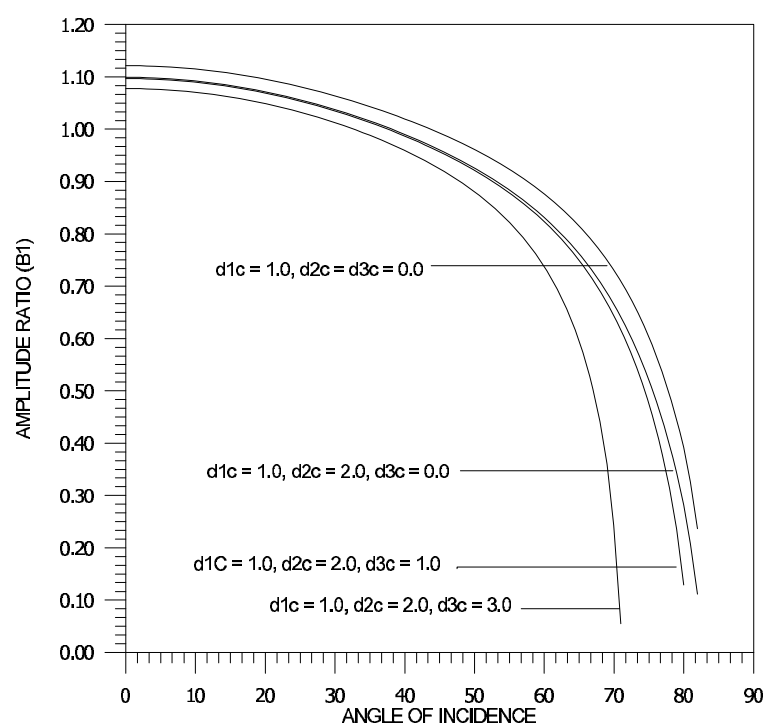

Fig. 16. Variations of Reflection coefficient for the first order approximation of the corrugation $\left(B_{1}\right)$ with $\gamma$ : when $\left(d_{1} c, d_{2} c, d_{3} c\right)=(1.0,0.0,0.0)$; $(1.0,2.0,0.0) ;(1.0,2.0,1.0)$; $(1.0,2.0,3.0)$.

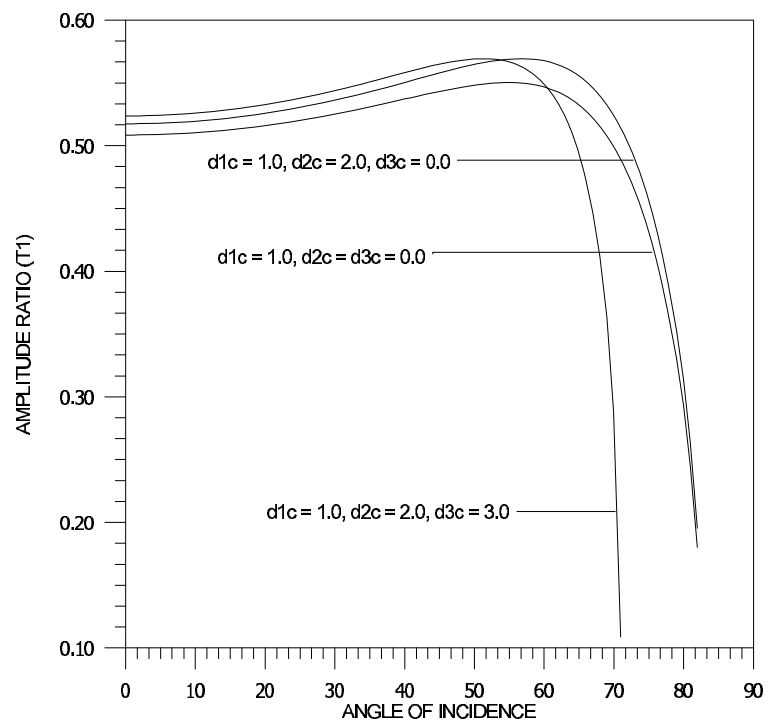

Fig. 17. Variations of the Transmission coefficient for the first order approximation of the corrugation $\left(T_{1}\right)$ with $\gamma$ : when $\left(d_{1} c, d_{2} c, d_{3} c\right)=(1.0,0.0,0.0) ; \quad(1.0,2.0,0.0) ;(1.0,2.0,1.0)$; $(1.0,2.0,3.0)$.

given in the Eq. (28), reduce to

$$
\begin{aligned}
& Q=\frac{\omega}{\beta_{h_{1}}} \cos \gamma, \quad U=\frac{\omega}{\beta_{h_{1}}}\left(\frac{\beta_{h_{1}}^{2}}{\beta_{h_{2}}^{2}}-\sin ^{2} \gamma\right)^{1 / 2}, \\
& Q_{n}=\frac{\omega}{\beta_{h_{1}}} \cos \gamma_{n}, \quad U_{n}=\frac{\omega}{\beta_{h_{1}}}\left(\frac{\beta_{h_{1}}^{2}}{\beta_{h_{2}}^{2}}-\sin ^{2} \gamma_{n}\right)^{1 / 2}, \\
& Q_{n}^{\prime}=\frac{\omega}{\beta_{h_{1}}} \cos \gamma_{n}^{\prime}, \quad U_{n}^{\prime}=\frac{\omega}{\beta_{h_{1}}}\left(\frac{\beta_{h_{1}}^{2}}{\beta_{h_{2}}^{2}}-\sin ^{2} \gamma_{n}^{\prime}\right)^{1 / 2} .
\end{aligned}
$$

The values of the coefficients given by Eqs. (36) and (37) for the plane interface become

$$
B=\frac{-U+m Q}{U+m Q}, \quad D=\frac{2 \sqrt{m} Q}{U+m Q} .
$$




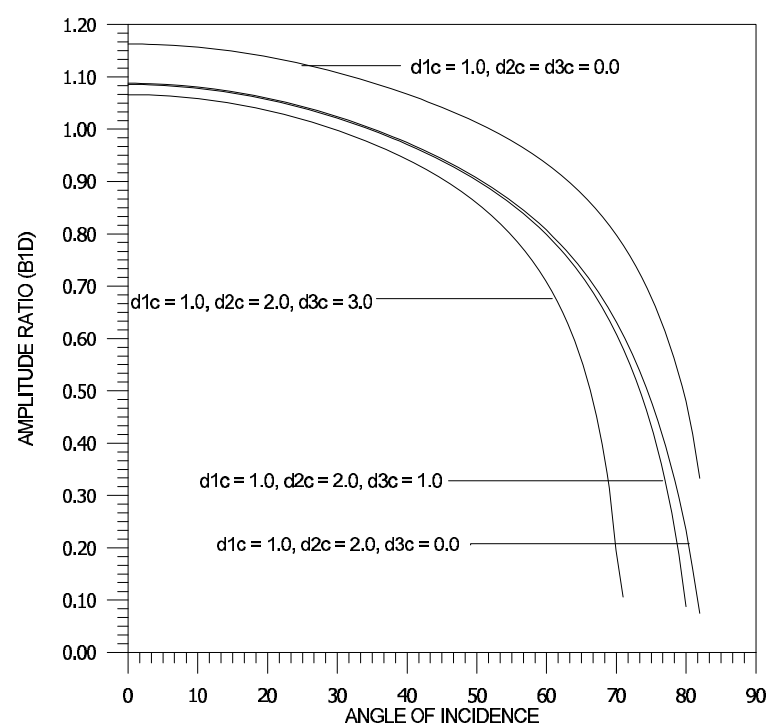

Fig. 18. Variations of the Reflection coefficient for the first order approximation of the corrugation $\left(B_{1}^{\prime}\right)$ with $\gamma$ : when $\left(d_{1} c, d_{2} c, d_{3} c\right)=(1.0,0.0,0.0) ; \quad(1.0,2.0,0.0) ;(1.0,2.0,1.0)$; $(1.0,2.0,3.0)$.

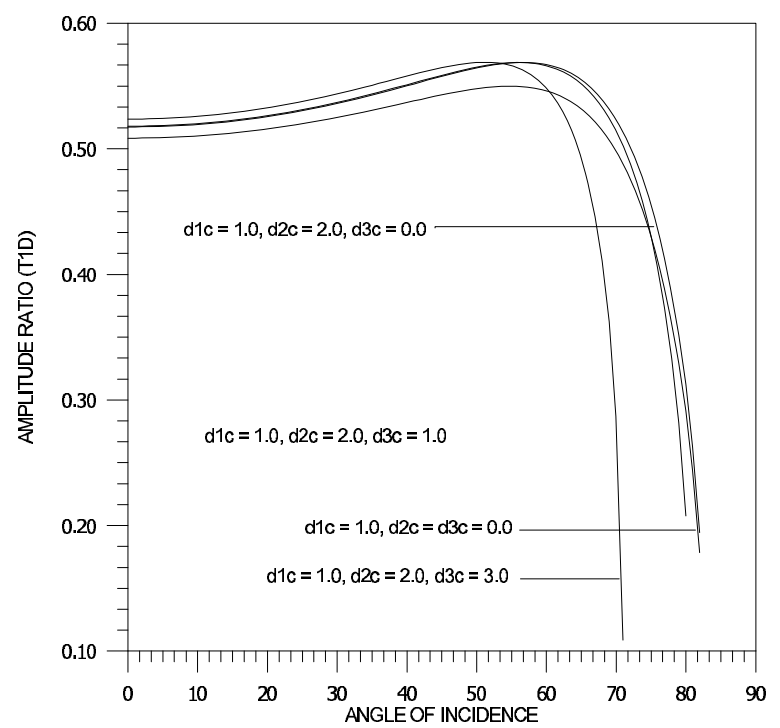

Fig. 19. Variations of the Transmission coefficient for the first order approximation of the corrugation $\left(T_{1}^{\prime}\right)$ with $\gamma$ : when $\left(d_{1} c, d_{2} c, d_{3} c\right)=(1.0,0.0,0.0) ;(1.0,2.0,0.0) ;(1.0,2.0,1.0)$; $(1.0,2.0,3.0)$.

The expressions of $B_{1}, D_{1}, B_{1}^{\prime}$ and $D_{1}^{\prime}$ in this case become

$$
\begin{aligned}
B_{1}=\Delta[ & m(1+B)\left(\frac{k^{*} \omega \sin \gamma}{\beta_{h_{1}}}-Q^{2}\right) \\
& +(1-B) Q U_{1} \\
& \left.+D \sqrt{m}\left(-U U_{1}+U^{2}-\frac{\left.k^{*} \omega \sin \gamma\right)}{\beta_{h_{1}}}\right)\right], \\
D_{1}=\Delta & {\left[\sqrt{m}(1+B)\left(\frac{k^{*} \omega \sin \gamma}{\beta_{h_{1}}}-Q^{2}\right)\right.} \\
& -D\left(\frac{k^{*} \omega \sin \gamma}{\beta_{h_{1}}}-U^{2}-m U Q_{1}\right)
\end{aligned}
$$

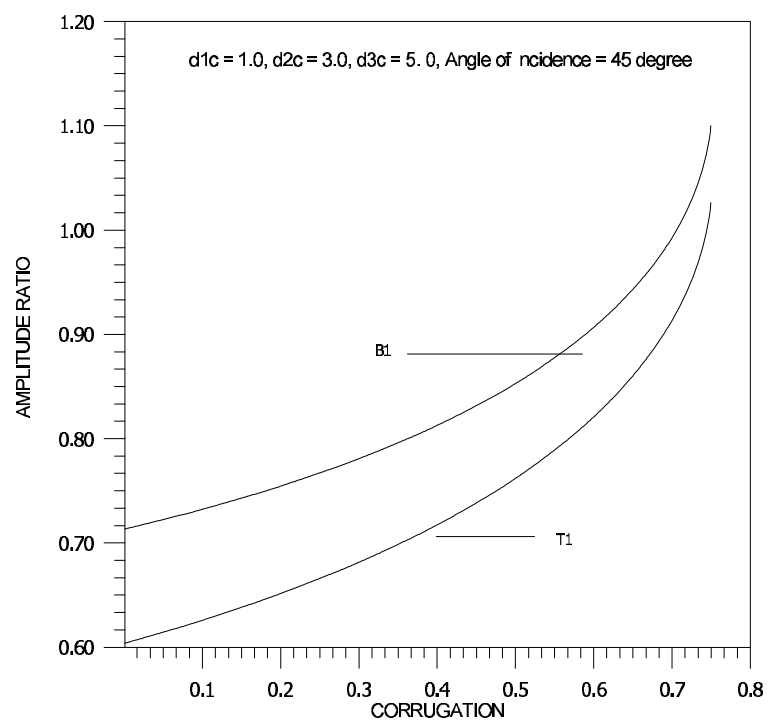

Fig. 20. Variations of Reflection and Transmission coefficients for the first order approximation of the corrugation $\left(B_{1}\right)$ and $\left(T_{1}\right)$ with $k^{*} c$ : when $d_{1} c=1.0, d_{2} c=3.0, d_{3} c=5.0$.

$$
\begin{aligned}
& \left.+\sqrt{m} Q Q_{1}(1-B)\right], \\
B_{1}^{\prime}=\Delta[ & -m(1+B)\left(\frac{k^{*} \omega \sin \gamma}{\beta_{h_{1}}}+Q^{2}\right) \\
& +(1-B) Q U_{1}^{\prime} \\
& \left.-D \sqrt{m}\left(U U_{1}^{\prime}-U^{2}-\frac{\left.k^{*} \omega \sin \gamma\right)}{\beta_{h_{1}}}\right)\right], \\
D_{1}^{\prime}=\Delta[ & -\sqrt{m}(1+B)\left(\frac{k^{*} \omega \sin \gamma}{\beta_{h_{1}}}+Q^{2}\right) \\
& +D\left(\frac{k^{*} \omega \sin \gamma}{\beta_{h_{1}}}+U^{2}+m U Q_{1}^{\prime}\right) \\
& \left.-\sqrt{m} Q Q_{1}^{\prime}(1-B)\right]
\end{aligned}
$$

where

$$
\Delta=\frac{\iota c}{2\left(U_{1}+m Q_{1}\right)} .
$$

These formulae give the reflection and refraction coefficients for the first order approximation of the corrugated interface between two uniform elastic half spaces. It can be verified that by removing the heterogeneity and anisotropy as explained above, the boundary conditions (29) and (30) match with those of Asano (1960).

\section{Numerical Results and Discussion}

In order to study the effect of inhomogeneity and anisotropy numerically on reflection and transmission coefficients, when a plane SH-wave become incident obliquely at a corrugated interface between two half spaces $H_{1}$ and $\mathrm{H}_{2}$, we have computed the modulus values of these coefficients for the model considered in section - Special case. For this purpose, we used the values of relevant parameters given as: For medium $H_{1}: N_{10}=38.5 \times 10^{11}$ dyne $/ \mathrm{cm}^{2}, M_{10}$ $=57.0 \times 10^{11}$ dyne $/ \mathrm{cm}^{2}, \rho_{1}=2.2 \mathrm{~g} / \mathrm{cm}^{3}$; For medium $H_{2}$ : $N_{20}=56.8 \times 10^{11}$ dyne $/ \mathrm{cm}^{2}, M_{20}=75.0 \times 10^{11}$ dyne $/ \mathrm{cm}^{2}$, 


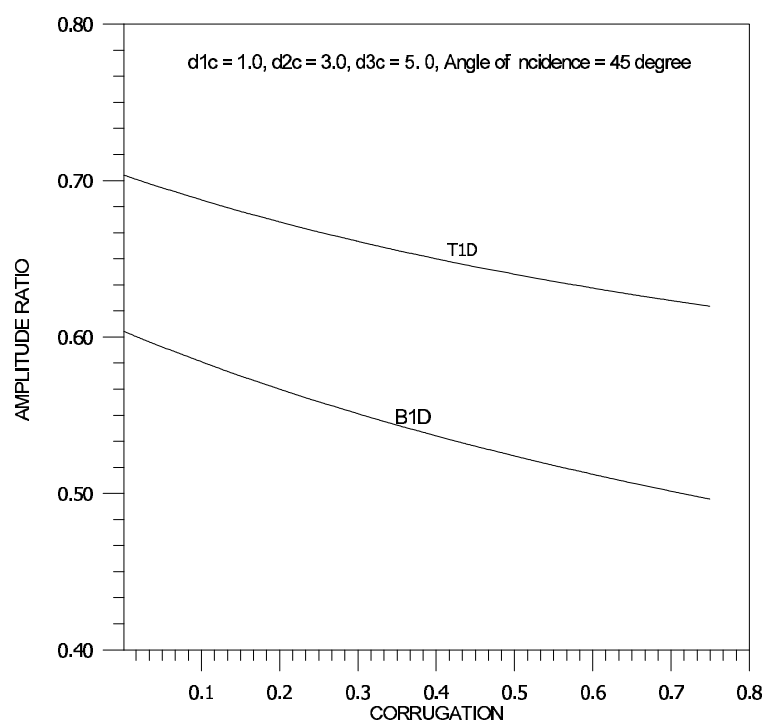

Fig. 21. Variations of the Reflection and Transmission coefficients for the first order approximation of the corrugation $\left(B_{1}^{\prime}\right)$ and $\left(T_{1}^{\prime}\right)$ with $k^{*} c$ : when $d_{1} c=1.0, d_{2} c=3.0, d_{3} c=5.0$ and $\gamma=45^{\circ}$.

$\rho_{2}=5.2 \mathrm{~g} / \mathrm{cm}^{3}$; and $\omega c / \beta_{h_{1}}=5.0, c$ being the amplitude of corrugation and $k^{*} c=0.00125$, wherever not mentioned. The non-dimensional quantities $d_{1} c$ and $d_{2} c$ represent the vertical heterogeneity factors in $H_{1}$ and $H_{2}$ respectively, while $d_{3} c$ is lateral heterogeneity factor in both media.

(i)Effect of the vertical heterogeneity: Figures 2 and 3 show the variations of reflection and transmission coefficients with respect to angle of incidence at a plane interface between $H_{1}$ and $H_{2}$. Here we have taken variations in vertical heterogeneity in $H_{1}$ only, and $H_{2}$ a homogeneous one, that is, $d_{2} c=d_{3} c=0$ and $d_{1} c=0.0,1.0,2.0,3.0$. The case $d_{1} c=d_{2} c=d_{3} c=0$ represent that both the media are homogeneous. We observe from Figs. 2 and 3 that there is a significant effect of the vertical heterogeneity $d_{1} c$, on the reflection and transmission coefficients. It is found that the reflection coefficient $B$ increases with $d_{1} c$, while the transmission coefficient $T$ decreases with $d_{1} c$.

Figures 4-7 depict the variations of reflection and transmission coefficients with respect to angle of incidence for the first- order approximation of the corrugated interface. The effect of $d_{1} c$ on these coefficients is clearly noticed. It is found that the values of reflection coefficients decreases with $d_{1} c$ while in the transmission coefficient, initially there is a little increase with $d_{1} c$ up to certain angle of incidence, beyond which it decreases with $d_{1} c$ significantly. However, the critical angles are different for different non-zero values of $d_{1} c$.

Figures $8-13$ show the effect of vertical heterogeneity in $H_{1}$ and $H_{2}$ (no lateral heterogeneity) on $B, T, B_{1}, T_{1}, B_{1}^{\prime}$ and $T_{1}^{\prime}$ respectively. The value of the vertical heterogeneity in medium $H_{1}$ is taken $d_{1} c=1.0$, a constant while the vertically heterogeneity $d_{2} c$, in medium $\mathrm{H}_{2}$ varies through values $0.0,3.0,5.0$ and 6.0. From Figs. 8 and 9, we note that the values of $B$ and $T$ increase with $d_{2} c$. The effect of $d_{2} c$ is maximum at $\gamma=0^{\circ}$ and minimum at $\gamma=82^{\circ}$, beyond which no reflection or transmission coefficient appear. In Fig. 10, the variation of reflection coefficient $B_{1}$ is

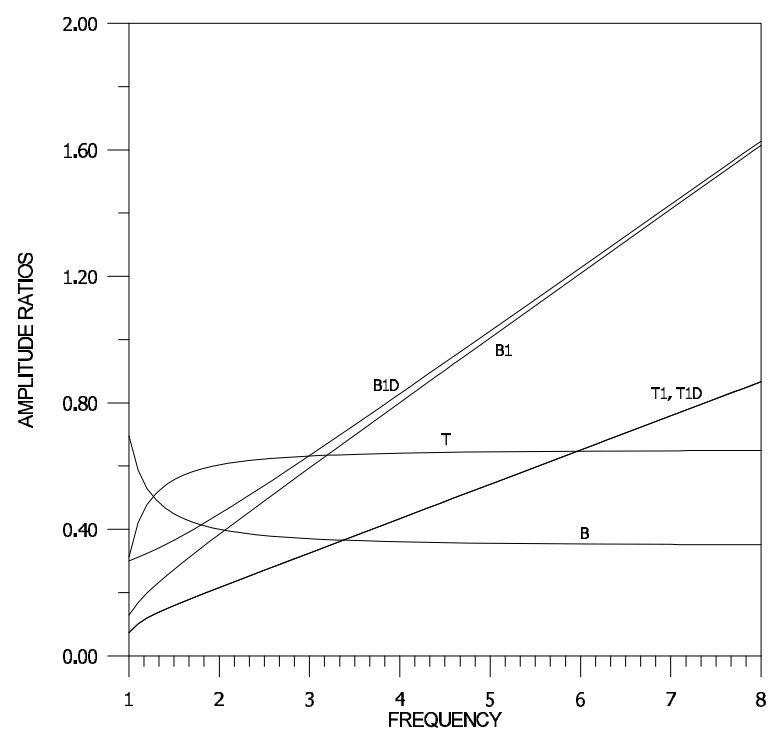

Fig. 22. Variations of the Reflection and Transmission coefficients for first order approximation of the corrugation $\left(B_{1}\right),\left(T_{1}\right),\left(B_{1}^{\prime}\right)$ and $\left(T_{1}^{\prime}\right)$ with frequency $\left(\omega c / \beta_{h_{1}}\right)$ : when $d_{1} c=1.0, d_{2} c=3.0, d_{3} c=5.0$ and $\gamma=45^{0}$.

different for different value of $\gamma$ and $d_{2} c$, while in Fig. 11, we note that the transmission coefficient $T_{1}$ decreases with $d_{2} c$. From Fig. 12, we note that when $d_{2} c=0.0$, the coefficient $B_{1}^{\prime}$ decreases slowly with $\gamma$, but as $d_{2} c$ takes values 3.0, 5.0 and 6.0 its value decreases up to certain angle and then start increases rapidly. In Fig. 13, we note that when $d_{2} c=0.0,3.0,5.0$ and 6.0 , the coefficient $T_{1}^{\prime}$ increases slowly up to certain value of $\gamma$ and then decreases rapidly with $\gamma$.

Figures 14 and 15 show the combined effect of lateral and vertical heterogeneities on the reflection and transmission coefficients versus angle of incidence, at the plane interface between $\mathrm{H}_{1}$ and $\mathrm{H}_{2}$. We can notice from these figures that for different values of $d_{1} c, d_{2} c$ and $d_{3} c$, the reflection coefficient $B$ takes minimumum value at $\gamma=0^{\circ}$ which increases further with $\gamma$, while transmission coefficient $T$ exhibit reverse behaviour.

Figures 16-19 show the effect of $d_{1} c, d_{2} c$ and $d_{3} c$ on $B_{1}, T_{1}, B_{1}^{\prime}$ and $T_{1}^{\prime}$. We notice from these figures that the values of the coefficients $B_{1}$ and $B_{1}^{\prime}$ takes maximum value at $\gamma=0^{\circ}$ and then decreases with increase of $\gamma$, while the values of the coefficients $T_{1}$ and $T_{1}^{\prime}$ behaves alike. The values of later coefficients first increase slightly from the values attained at $\gamma=0^{\circ}$, then achieve maximum at certain angle of incidence and then decrease sharply. Also, the effect of $d_{3} c$ is found more dominantant beyond $\gamma=50^{\circ}$ angle of incidence.

(ii)Effect of corrugation: Firstly, it is to be noted that the coefficients $B$ and $T$ do not depend on the corrugation parameter $k^{*} c$, as they are the reflection and transmission coefficients respectively for the plane interface. To study the effect of $k^{*} c$ on $B_{1}, T_{1}, B_{1}^{\prime}$ and $T_{1}^{\prime}$, we have computed them for very small values of $k^{*} c$. The reason is, because this method is not applicable for large values of corrugation. Here we have found that these coefficients are strongly effected by the corrugation $k^{*} c$. Figure 20 shows the variation of $B_{1}$ and $T_{1}$ 


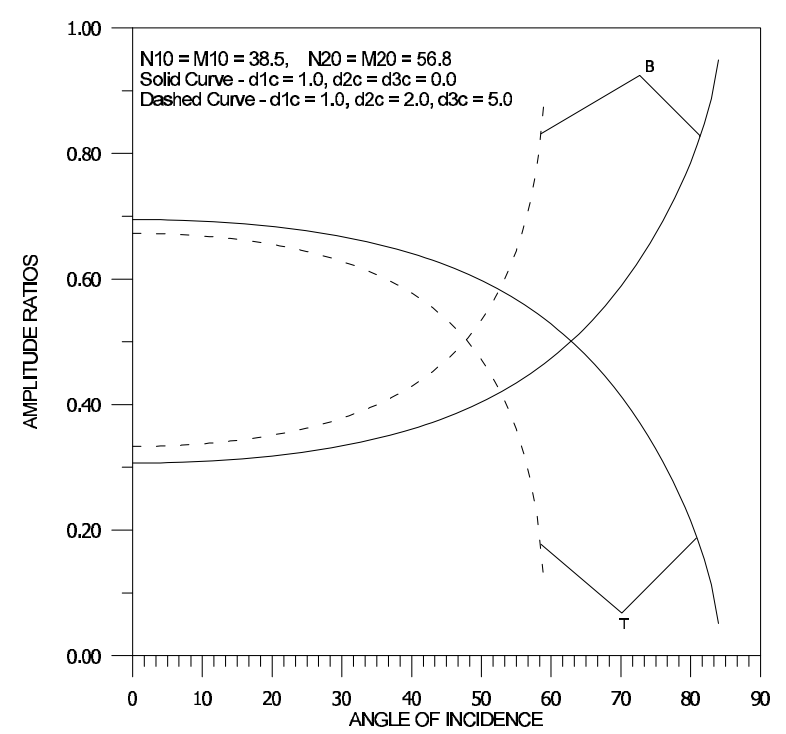

Fig. 23. Variations of the Reflection and Transmission coefficients at the plane interface (B) and (T) with $\gamma$ : when $N_{10}=38.5=M_{10}, N_{20}=56.8=M_{20}$ and $d_{1} c=1.0,\left(d_{2} c, d_{3} c\right)=(0,0) ;(2.0,5.0)$.

versus corrugation $\left(k^{*} c\right)$ for $d_{1} c=1.0, d_{2} c=3.0, d_{3} c=$ 5.0, $\omega c / \beta_{h_{1}}=5.0$ and $\gamma=45^{\circ}$. We notice that the values of $B_{1}$ and $T_{1}$ increase monotonically with the increase of $k^{*} c$. Figure 21 shows the variation of $B_{1}^{\prime}$ and $T_{1}^{\prime}$ versus $k^{*} c$ with same values of parameters as taken for Fig. 20. We notice from this figure that the values of $B_{1}^{\prime}$ and $T_{1}^{\prime}$ decrease with $k^{*} c$. Also, we observe from these figures that the values of $B_{1}$ and $T_{1}$ are more than those of $B_{1}^{\prime}$ and $T_{1}^{\prime}$ as $k^{*} c$ takes values through 0.0 to 0.8 .

(iii) Effect of frequency: To study the effect of frequency of incident wave on reflection and transmission coefficients for both plane and corrugated interfaces, we fix the value of parameters as $d_{1} c=1.0, d_{2} c=3.0, d_{3} c=5.0$ and $\gamma=$ $45^{\circ}$. Figure 22 shows that, these coefficients are influenced by the frequency $\left(\omega c / \beta_{h_{1}}\right)$ of the incident $\mathrm{SH}$-wave. The values of relection and transmission coefficients $B$ and $T$ decrease and increase respectively with $\omega c / \beta_{h_{1}}$ in the range $1.0<\omega c / \beta_{h_{1}}<1.6$ and thereafter they become constant for large values of $\omega c / \beta_{h_{1}}$. The coefficients for first order approximation of corrugation $B_{1}, B_{1}^{\prime}, T_{1}$ and $T_{1}^{\prime}$ increase with $\left(\omega c / \beta_{h_{1}}\right)$.

(iv) Effect of heterogeneity and anisotropy: Figures 23 and 24 show the variation of $B, T$ and $B_{1}, T_{1}, B_{1}^{\prime}, T_{1}^{\prime}$ with $\gamma$ respectively, when both media are free from anisotropy and lateral heterogeneity, while vertical heterogeneity is present in $H_{1}$ only. From Fig. 23, we note that when anisotropy of both media are removed and the vertical heterogeneity in $H_{1}$ is fixed, the values of the reflection coefficient $B$ takes minimum value at $\gamma=0^{\circ}$ and increases with $\gamma$, while the values of the transmission coefficient $T$ possesses reverse behaviour. But, when the vertical heterogeneity is $d_{2} c=2.0$ and lateral heterogeneity is $d_{3} c=5.0$, the values of the coefficient $B$ are greater and the values of $T$ are less in comarision to the case of when $d_{2} c=d_{3} c=0.0$. This shows that the lateral and vertical heterogeneities have significant effect on the reflection and transmission coefficients at plane

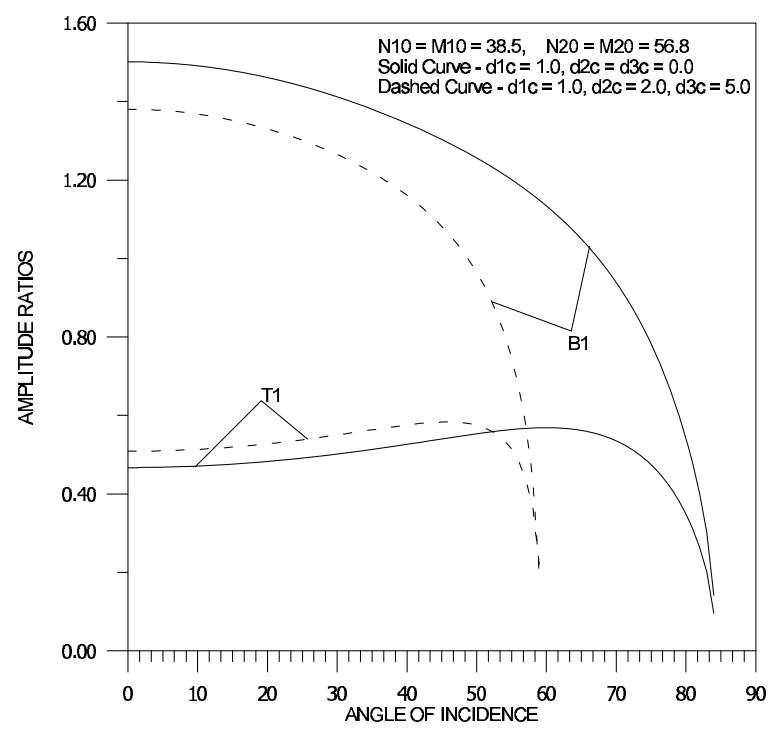

Fig. 24. Variations of the Reflection and Transmission coefficients for the first order approximation of the corrugation $\left(B_{1}\right),\left(T_{1}\right),\left(B_{1}^{\prime}\right)$ and $\left(T_{1}^{\prime}\right)$ with $\gamma$ : when $N_{10}=38.5=M_{10}, N_{20}=56.8=M_{20}$ and $d_{1} c=1.0$, $\left(d_{2} c, d_{3} c\right)=(0,0):(2.0,5.0)$.

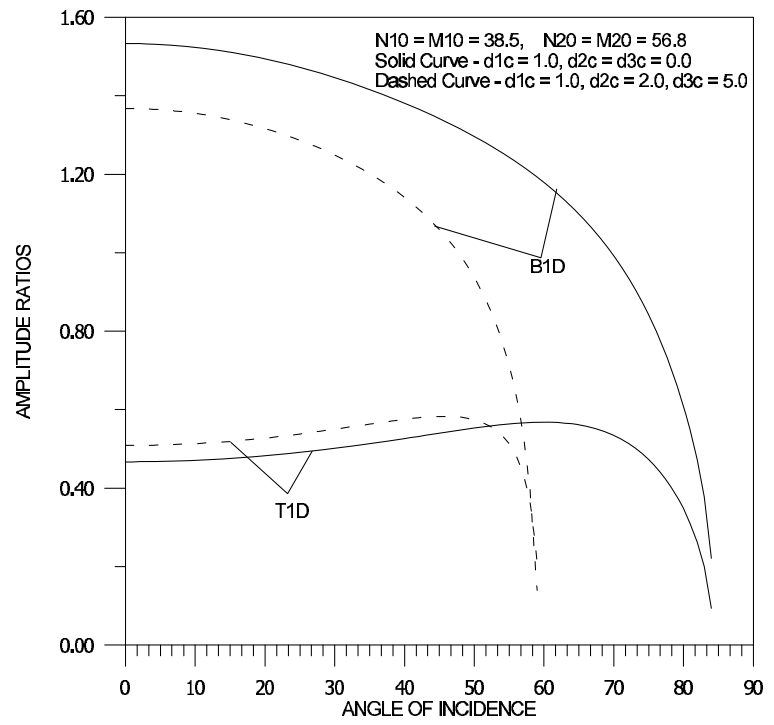

Fig. 25. Variations of the Reflection and Transmission coefficients for the first order approximation of the corrugation $\left(B_{1}\right),\left(T_{1}\right),\left(B_{1}^{\prime}\right)$ and $\left(T_{1}^{\prime}\right)$ with $\gamma$ : when $N_{10}=38.5=M_{10}, N_{20}=56.8=M_{20}$ and $d_{1} c=1.0$, $\left(d_{2} c, d_{3} c\right)=(0,0) ;(2.0,5.0)$.

interface.

Figure 24 shows the variation of reflection and transmission coefficients for first order approximation of corrugation with $\gamma$, when anisotropy and lateral heterogeniety is removed and the vertical heterogeneity in $H_{1}$ and $H_{2}$ are taken as $d_{1} c=1.0$ and $d_{2} c=0.0$ respectively. We notice that the values of coefficient $T_{1}$ are less than the values of coefficient $B_{1}$. The same is true when the values $d_{2} c=2.0$ and $d_{3} c=5.0$ are introduced. A similar behaviour of coefficients $B_{1}^{\prime}$ and $T_{1}^{\prime}$ is observed from Fig. 25.

Figures 26-31, show the variation of $B, T, B_{1}, T_{1}, B_{1}^{\prime}$ and $T_{1}^{\prime}$ with $\gamma$ for different values of the anisotropy factor in both media. These values are taken as $N_{10} / M_{10}=1.0,0.5,0.7$ in $H_{1}$ and $N_{20} / M_{20}=1.0,0.8,0.9$ in $H_{2}$, while the hetero- 


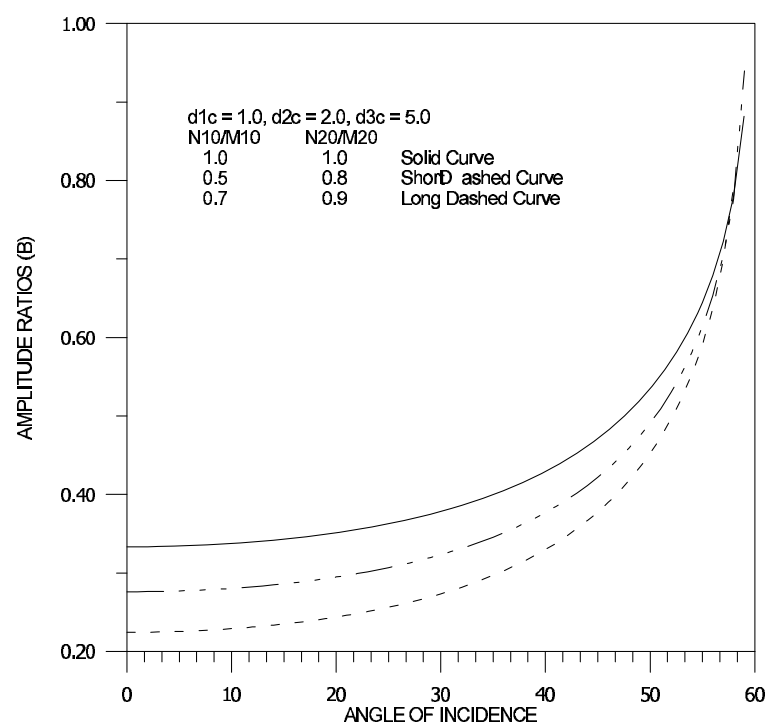

Fig. 26. Variations of the Reflection coefficient at the plane interface (B) with $\gamma$ : for $\left(N_{10} / M_{10}, N_{20} / M_{20}\right)=(1.0,1.0)$; $(0.5,0.8) ;(0.7,0.9)$.

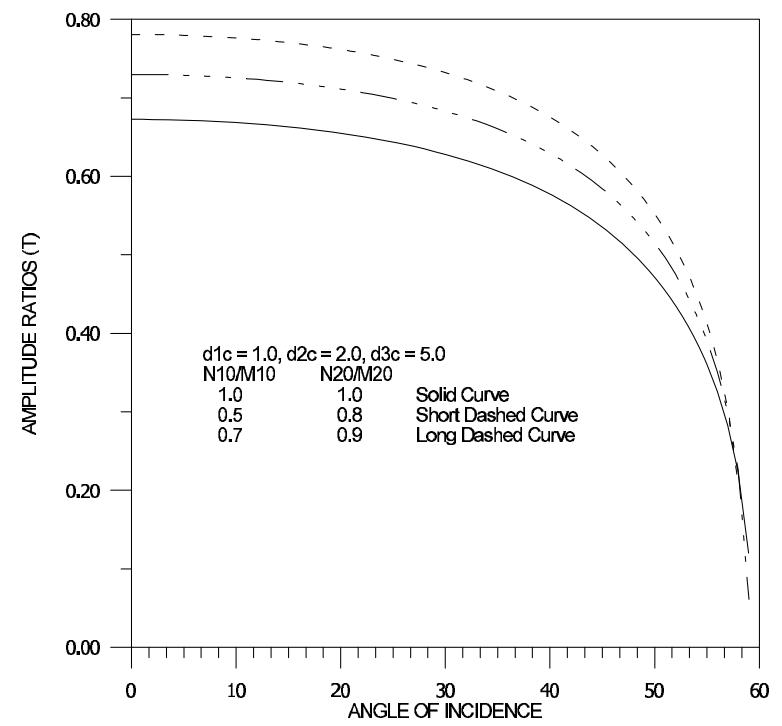

Fig. 27. Variations of the Transmission coefficient at the plane interface $(\mathrm{T})$ with $\gamma$ : for $\left(N_{10} / M_{10}, N_{20} / M_{20}\right)=(1.0,1.0) ;(0.5,0.8) ;(0.7,0.9)$.

geneities are taken as $d_{1} c=1.0, d_{2} c=2.0, d_{3} c=5.0$. The case $N_{10} / M_{10}=N_{20} / M_{20}=1.0$ correspond to isotropy of both the media. We notice from these figures that each coefficient, whether on the plane interface or on the corrugated interface, is significantly effected by the anisotropy of the media. The reflection and transmission coefficients for plane interface possess reverse behaviour while other coefficients behave alike.

\section{Conclusions}

Using the Rayleigh's method, the formulae for reflection and transmission coefficients due to incident SH-wave at a corrugated interface between two laterally and vertically heterogeneous transversely isotropic elastic solid half spaces have been obtained in closed form. We conclude that

(1) Reflection and transmission coefficients for the first order approximation of the corrugation given by (43) with

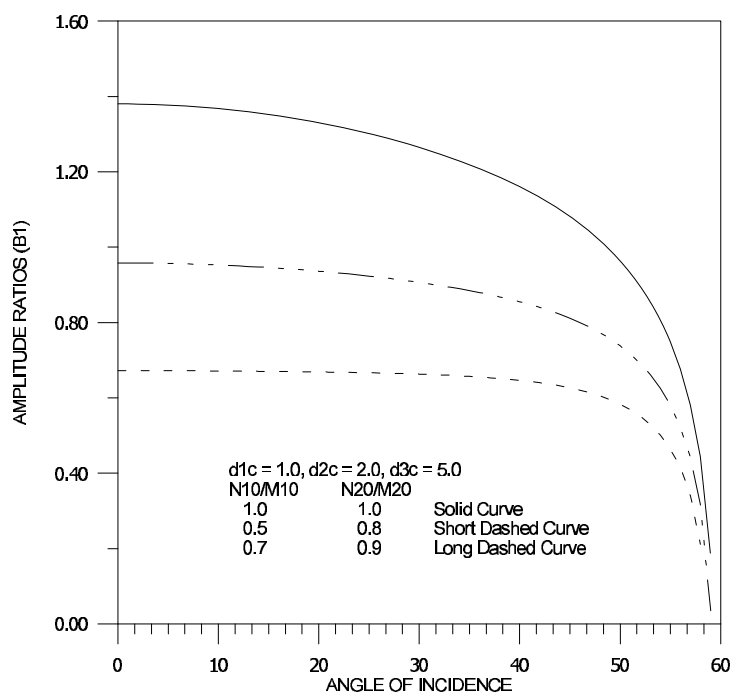

Fig. 28. Variations of the Reflection coefficient for the first order approximation of the corrugation $\left(B_{1}\right)$ with $\gamma$ : when $\left(N_{10} / M_{10}, N_{20} / M_{20}\right)=(1.0,1.0) ;(0.5,0.8) ;(0.7,0.9)$.

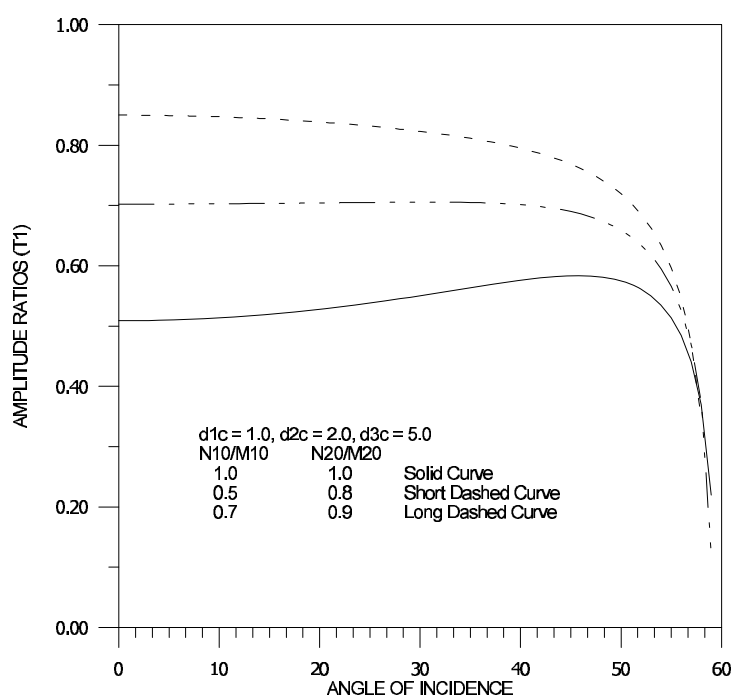

Fig. 29. Variations of the Transmission coefficient for the first order approximation of the corrugation $\left(T_{1}\right)$ with $\gamma$ : when $\left(N_{10} / M_{10}, N_{20} / M_{20}\right)=(1.0,1.0) ;(0.5,0.8) ;(0.7,0.9)$.

(44) depend on $\zeta_{-n}$ and $\zeta_{n}$ i.e. on $\zeta$, the corrugation of the interface. In the special case considered, if we remove the corrugation of the interface by putting $c=0$ into the formulae given in Appendix-II, then $B_{1}=T_{1}=B_{1}^{\prime}=$ $T_{1}^{\prime}=0$ and we have only the non-vanishing expressions of $B$ and $T$, given by (46) and (47), which are the reflection and transmission coefficients at the plane interface between two anisotropic homogeneous half-spaces.

(2) From the formulae (36)-(37) and (43) and our numerical results, we also conclude that the reflection and transmission coefficients strongly depend upon heterogeneity, anisotropy, frequency and angle $\gamma$ of the incident SHwave.

(i) Reflection and transmission coefficients for the first order approximation of the corrugation are found to be effected by the vertical heterogeneity factor $d_{1} c$. This effect is found least near normal incidence, when $d_{2} c$ and $d_{3} c$ are absent and 


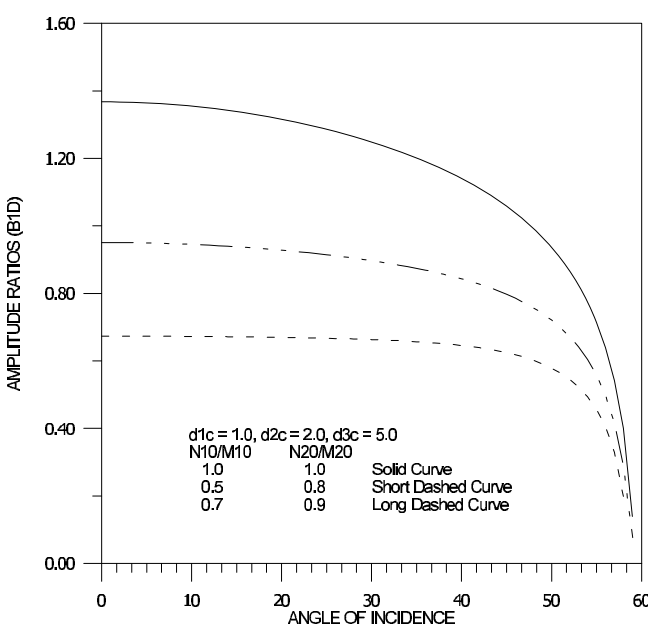

Fig. 30. Variations of the Reflection coefficient for the first order approximation of the corrugation $\left(B_{1}^{\prime}\right)$ with $\gamma$ : when $\left(N_{10} / M_{10}, N_{20} / M_{20}\right)=(1.0,1.0) ;(0.5,0.8) ;(0.7,0.9)$.

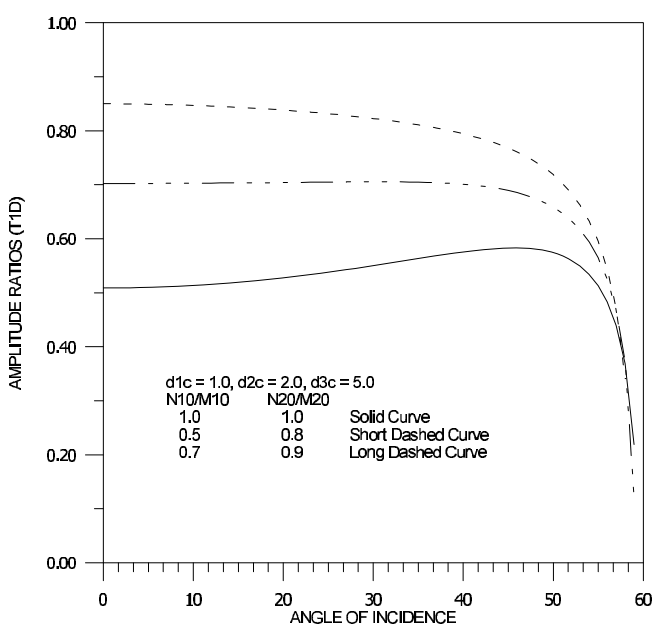

Fig. 31. Variations of the Transmission coefficient for the first order approximation of the corrugation $\left(T_{1}^{\prime}\right)$ with $\gamma$ : when $\left(N_{10} / M_{10}, N_{20} / M_{20}\right)=(1.0,1.0) ;(0.5,0.8) ;(0.7,0.9)$.

$k^{*} c$ is very small.

(ii) In the case when $d_{1} c$ is fixed and $d_{2} c$ is varied through the values $0.0,3.0,5.0$ and 6.0 then the reflection and transmission coefficients are found to be influenced more by $d_{2} c$ at $\gamma=0^{\circ}$.

(iii) As the corrugation increases, the values of $B_{1}$ and $T_{1}$ increase slowly while the values of $B_{1}^{\prime}$ and $T_{1}^{\prime}$ decrease slowly. The values of coefficients $B$ and $T$ remain uneffected.

(iv) When both the media are anisotropic and heterogeneous, these amplitude ratios are found to be influenced by frequency of the incident wave. For larger values of frequency, the values of the coefficients $B$ and $T$ remain almost constant.

(v) When lateral heterogeneity is removed and $d_{1} c=$ $1.0, d_{2} c=0.0$, then the values of $B$ increase with $\gamma$, while the values of $T$ decrease with $\gamma$. On the other hand the remaining amplitude ratios decreases rapidly with $\gamma$. The introduction of lateral heterogeneity do not allow these coefficients to occur at and beyond $\gamma=60^{\circ}$. (vi) The problems of Asano (1960), Gupta (1987) and Tomar and Saini (1997) have been obtained as a particular cases. Some new results have also been presented.

\section{Appendix A.}

$$
\begin{aligned}
& \Delta_{B_{1}}=\frac{\iota c}{2}\left[(1+B)\left\{m\left(\frac{k^{*} \omega \sin \gamma}{\beta_{h_{1}}}-\frac{d_{1}^{2}}{4}-Q^{2}\right)+\frac{d_{2}^{2}}{4}\right\}\right. \\
& +(1-B) Q U_{1}+D \sqrt{m}\left(-U U_{1}-\frac{d_{1} d_{2}}{4}\right. \\
& \left.-\frac{k^{*} \omega \sin \gamma}{\beta_{h_{1}}}+\frac{d_{2}^{2}}{4}+U^{2}\right) \\
& +\iota\left\{(1+B)\left(\frac{U_{1} d_{2}}{2}-\frac{m d_{3} k^{*}}{2}\right)-(1-B) \frac{Q d_{2}}{2}\right. \\
& \left.\left.+D \sqrt{m}\left(\frac{U d_{2}}{2}-\frac{U_{1} d_{1}}{2}+\frac{k^{*} d_{3}}{2}\right)\right\}\right], \\
& \Delta_{D_{1}}=\frac{\iota c}{2}\left[(1+B) \sqrt{m}\left(\frac{k^{*} \omega \sin \gamma}{\beta_{h_{1}}}-\frac{d_{1}^{2}}{4}-Q^{2}+\frac{d_{1} d_{2}}{4}\right)\right. \\
& -\sqrt{m}(1-B) Q Q_{1} \\
& +D\left(U^{2}+\frac{d_{2}^{2}}{4}-\frac{k^{*} \omega \sin \gamma}{\beta_{h_{1}}}-\frac{m d_{1}^{2}}{4}+m U Q_{1}\right) \\
& +\iota\left\{(1+B) \sqrt{m}\left(-\frac{Q_{1} d_{2}}{2}-\frac{d_{3} k^{*}}{2}\right)\right. \\
& -\sqrt{m}(1-B) \frac{Q d_{1}}{2} \\
& \left.\left.+D\left(\frac{k^{*} d_{3}}{2}+\frac{m U d_{1}}{2}+\frac{m Q_{1} d_{1}}{2}\right)\right\}\right] \text {, } \\
& \Delta_{B_{1}^{\prime}}=\frac{\iota c}{2}\left[(1+B)\left\{-m\left(\frac{k^{*} \omega \sin \gamma}{\beta_{h_{1}}}+\frac{d_{1}^{2}}{4}+Q^{2}\right)+\frac{d_{2}^{2}}{4}\right\}\right. \\
& +(1-B) Q U_{1}^{\prime}-\sqrt{m} D \\
& \cdot\left(U U_{1}^{\prime}+\frac{d_{1} d_{2}}{4}-\frac{k^{*} \omega \sin \gamma}{\beta_{h_{1}}}-\frac{d_{2}^{2}}{4}-U^{2}\right) \\
& +\iota\left\{(1+B)\left(\frac{U_{1}^{\prime} d_{2}}{2}+\frac{m d_{3} k^{*}}{2}\right)\right. \\
& -(1-B) \frac{Q d_{2}}{2} \\
& \left.\left.-\sqrt{m} D\left(\frac{U_{1}^{\prime} d_{1}}{2}-\frac{U d_{2}}{2}+\frac{k^{*} d_{3}}{2}\right)\right\}\right], \\
& \Delta_{D_{1}^{\prime}}=\frac{\iota c}{2}\left[-(1+B) \sqrt{m}\left(\frac{k^{*} \omega \sin \gamma}{\beta_{h_{1}}}+\frac{d_{1}^{2}}{4}+Q^{2}-\frac{d_{1} d_{2}}{4}\right)\right. \\
& -\sqrt{m}(1-B) Q Q_{1}^{\prime} \\
& +D\left(U^{2}+\frac{d_{2}^{2}}{4}+\frac{k^{*} \omega \sin \gamma}{\beta_{h_{1}}}-\frac{m d_{1}^{2}}{4}+m U Q_{1}^{\prime}\right) \\
& +\iota\left\{(1+B) \sqrt{m}\left(\frac{d_{3} k^{*}}{2}-\frac{Q_{1}^{\prime} d_{2}}{2}\right)\right. \\
& -\sqrt{m}(1-B) \frac{Q d_{1}}{2} \\
& \left.\left.-D\left(\frac{k^{*} d_{3}}{2}-\frac{m U d_{1}}{2}-\frac{m Q_{1}^{\prime} d_{1}}{2}\right)\right\}\right], \\
& \Delta_{1}=\left(U_{1}+m Q_{1}\right)+\frac{\iota}{2}\left(d_{1} m-d_{2}\right), \\
& \Delta_{1}^{\prime}=\left(U_{1}^{\prime}+m Q_{1}^{\prime}\right)+\frac{\iota}{2}\left(d_{1} m-d_{2}\right) .
\end{aligned}
$$




\section{Appendix B.}

$$
\begin{aligned}
& B_{1}=\frac{l c}{2\left(R_{1}+\frac{M_{10}}{M_{20}} Q_{1}\right)} \\
& \text {. }\left[\frac { R _ { 1 } \omega } { \beta _ { h _ { 1 } } } \left\{(1-B) \sqrt{\frac{N_{10}}{M_{10}}} \cos \gamma\right.\right. \\
& \left.-D \sqrt{\frac{M_{10}}{M_{20}}} \sqrt{\frac{N_{20}}{M_{20}}\left(\frac{\beta_{h_{1}}^{2}}{\beta_{h_{2}}^{2}}-\sin ^{2} \gamma\right)}\right\} \\
& +\frac{M_{10}}{M_{20}}(1+B)\left(\frac{k^{*} \omega \sin \gamma}{\beta_{h_{1}}}-Q^{2}\right) \\
& \left.-D \sqrt{\frac{M_{10}}{M_{20}}}\left(\frac{k^{*} \omega \sin \gamma}{\beta_{h_{1}}}-R^{2}\right)\right], \\
& D_{1}=\frac{\iota c}{2\left(R_{1}+\frac{M_{10}}{M_{20}} Q_{1}\right)} \\
& \cdot\left[\sqrt{\frac{M_{10}}{M_{20}}}(1+B)\left(\frac{k^{*} \omega \sin \gamma}{\beta_{h_{1}}}-Q^{2}\right)\right. \\
& -D\left(\frac{k^{*} \omega \sin \gamma}{\beta_{h_{1}}}-R^{2}\right) \\
& +\sqrt{\frac{M_{10}}{M_{20}}} \frac{Q_{1} \omega}{\beta_{h_{1}}} \\
& \cdot\left\{D \sqrt{\frac{M_{10}}{M_{20}}} \sqrt{\frac{N_{20}}{M_{20}}\left(\frac{\beta_{h_{1}}^{2}}{\beta_{h_{2}}^{2}}-\sin ^{2} \gamma\right)}\right. \\
& \left.\left.-(1-B) \sqrt{\frac{N_{10}}{M_{10}}} \cos \gamma\right\}\right], \\
& B_{1}^{\prime}=\frac{\iota c}{2\left(R_{1}^{\prime}+\frac{M_{10}}{M_{20}} Q_{1}^{\prime}\right)} \\
& \text {. }\left[\frac { R _ { 1 } ^ { \prime } \omega } { \beta _ { h _ { 1 } } } \left\{(1-B) \sqrt{\frac{N_{10}}{M_{10}}} \cos \gamma\right.\right. \\
& \left.-D \sqrt{\frac{M_{10}}{M_{20}}} \sqrt{\frac{N_{20}}{M_{20}}\left(\frac{\beta_{h_{1}}^{2}}{\beta_{h_{2}}^{2}}-\sin ^{2} \gamma\right)}\right\} \\
& -\frac{M_{10}}{M_{20}}(1+B)\left(\frac{k^{*} \omega \sin \gamma}{\beta_{h_{1}}}+Q^{2}\right) \\
& \left.+D \sqrt{\frac{M_{10}}{M_{20}}}\left(\frac{k^{*} \omega \sin \gamma}{\beta_{h_{1}}}+R^{2}\right)\right], \\
& D_{1}^{\prime}=\frac{\iota c}{2\left(R_{1}^{\prime}+\frac{M_{10}}{M_{20}} Q_{1}^{\prime}\right)} \\
& \cdot\left[-\sqrt{\frac{M_{10}}{M_{20}}}(1+B)\left(\frac{k^{*} \omega \sin \gamma}{\beta_{h_{1}}}+Q^{2}\right)\right. \\
& +D\left(\frac{k^{*} \omega \sin \gamma}{\beta_{h_{1}}}+R^{2}\right) \\
& -\sqrt{\frac{M_{10}}{M_{20}}} \frac{Q_{1}^{\prime} \omega}{\beta_{h_{1}}} \\
& \cdot\left\{(1-B) \sqrt{\frac{N_{10}}{M_{10}}} \cos \gamma\right.
\end{aligned}
$$

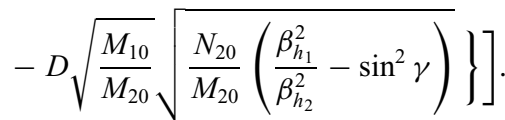

Acknowledgments. The authors are highly thankful to the referees, Professor Hiroshi Takenaka and Professor Rafael A. Benites for critically examining the paper and valuable suggestions which had led to significant improvements in the paper.

\section{References}

Abubakar, I., Scattering of plane elastic waves at rough surfaces, Proc. Camb. Phil. Soc., 58, 136-157, 1962a.

Abubakar, I., Reflection and refraction of plane SH-waves at irregular interfaces, J. Phys. Earth, 10, 1-14, 1962 b.

Abubakar, I., Reflection and refraction of plane SH-waves at irregular interfaces, J. Phys. Earth, 10, 15-20, 1962c.

Acharya, H. K., Reflection from the free surface of an inhomogeneous media, Bull. Seismol. Soc. Am., 60, 1101-1104, 1970.

Adams, W. M. and Chung-Po-Chang, Wave propagation phenomenon at an irregular infinite interface, Part I, Bull. Seismol. Soc. Am., 54, 2209-2222, 1964.

Aki, K. and K. L. Larner, Surface motion of layered medium having an irregular interface due to incident plane SH-waves, J. Geophys. Res., 75, 933-954, 1970.

Asano, S., Reflection and refraction of elastic waves at a corrugated boundary surface, Part I, The case of incidence of SH-wave, Bull. Earthq. Res. Inst., 38, 117-197, 1960 .

Asano, S., Reflection and refraction of elastic waves at a corrugated boundary surface, Part II, Bull. Earthq. Res. Inst., 39, 367-466, 1961.

Asano, S., Reflection and refraction of elastic waves at a corrugated interface, Bull. Seismol. Soc. Am., 56, 201-221, 1966.

Chattopadhyay, A. and A. Pal, Propagation of SH-waves in an inhomogeneous medium with irregular interface lying over an initially stressed elastic half space, Bul. Inst. Politeh. 'Gheorghe Gheorghiu-Dej', Bucuresti, Ser. Electroteh., 44(3), 47-56, 1982.

Daley, P. F. and F. Hron, Reflection and transmission coefficients for transversely isotropic media, Bull. Seism. Soc. Am., 67(3), 661-675, 1977.

Dunkin, J. W. and A. C. Eringen, The reflection of elastic waves from the wavy boundary of a half-space, Proceedings of the 4th U. S. National Congress on Applied Mechanics, University of California Press, Berkeley, 143-160, 1962.

Gilbert, F. and L. Knopoff, Seismic scattering from topographic irregularities, J. Geophys. Res., 65, 3437-3444, 1960.

Gupta, R. N., Reflection of plane waves from a linear transition layer in liquid media, Geophysics, 30, 122-132, 1965.

Gupta, S. K., Reflection and refraction from curved interfaces: model-study, Geophys. Prospect., 26, 82-96, 1978.

Gupta, S., Reflection and transmission of SH-waves in laterally and vertically heterogeneous media at an irregular boundary, Geophysical Transactions, 33(2), 89-111, 1987.

Henneke, E. G., Reflection-refraction of stress waves at a plane boundary between anisotropic media, J. Acoust. Soc. Am., 51, 210-217, 1972.

Keith, C. M. and S. Crampin, Seismic body waves in anisotropic media: Reflection and refraction at a plane interface, Geophys. J. Roy. Astron Soc., 49, 181-208, 1977.

Lay, T. and T. C. Wallace, Modern Global Seismology, Academic, San Diego, 1995

Malhotra, S., S. D. Chopra, and M. L. Gogna, Reflection and transmission of $\mathrm{SH}$-waves from a laterally and vertically heterogeneous transition layer of finite thickness, Acta Geophysica Polonica, 30, 341-357, 1982.

Mandal, B., Reflection and transmission properties of elastic wave on a plane interface for general anisotropic media, J. Acoust. Soc. Am., 90, 1106-1118, 1991.

Musgrave, M. J. P., Reflection and refraction of plane elastic waves at a plane boundary between aelotropic media, Geophys. J. Roy. Astron. Soc., 2, 406-418, 1960

Rokhlin, S. I., T. K. Bolland, and L. Adler, Reflection and refraction of elastic waves on a plane interface between two generally anisotropic media, J. Acoust. Soc. Am., 79, 906-918, 1986.

Ruger, A., P-wave reflection coefficients for transversely isotropic models with vertical and horizontal axis of symmetry, Geophysics, 62, 713-722, 1997.

Saini, S. L. and S. J. Singh, Effect of anisotropy on the reflection of SHwaves at an interface, Geophys. Res. Bull., 15(2), 67-73, 1977a.

Saini, S. L. and S. J. Singh, Reflection of SH-waves from an inhomogeneous half-space, Geophys. Res. Bull., 15(3), 1-12, 1977b

Sato, R., The reflection of elastic waves on corrugated surfaces, J. Seismol. Soc. Jpn., 8, 8-22, 1955.

Shearer, P. M., Introduction to Seismology, Cambridge University Press, 
Cambridge, 1999.

Sheriff, R. E. and L. P. Geldart, Exploration Seismology, 2nd ed., Cambridge University Press, Cambridge, 1995.

Singh, B. M., S. J. Singh, S. D. Chopra, and M. L. Gogna, On Love waves in laterally and vertically heterogeneous layered media, Geophys. J. Roy. Astron. Soc., 45, 357-370, 1976.

Singh, B. M., S. J. Singh, and S. D. Chopra, Reflection and refraction of $\mathrm{SH}$-waves at the plane boundary between two laterally and vertically heterogeneous solids, Acta Geophys. Polonica, 36, 209-216, 1978.

Slavin, L. M. and B. Wolf, Scattering of Love waves in a surface layer with an irregular boundary for the case of a rigid underlying half space, Bull. Seismol. Soc. Am., 60(3), 859-877, 1970.

Sokolnikoff, I. S., Mathematical Theory of Elasticity, McGraw-Hill, NY, 1956.

Sumner, J. H. and H. Deresiewicz, Waves in an elastic plate with an irregular boundary, PAGEOPH, 96, 106-125, 1972.

Tomar, S. K. and S. L. Saini, Reflection and refraction of SH-waves at a corrugated interface between two dimensional transversely isotropic half spaces, J. Phys. Earth, 45, 347-362, 1997.

Tomar, S. K., R. Kumar, and A. Chopra, Reflection/refraction of SH-waves at corrugated interface between transversely isotropic and visco-elastic solid half-spaces, Acta Geophysica Polonica, 50, 231-249, 2002.

Yamada, T. and R. Sato, SH-wave propagation in medium having stepshaped discontinuity, J. Phys. Earth, 24, 105-130, 1976.

Zhang, R. C., L. Y. Zhang, and M. Shinozuka, Seismic waves in a laterally inhomogeneous layered medium: Theory, J. Appl. Mech., 64, 50-58, 1997.

S. K. Tomar (e-mail: sktomar@yahoo.com) and J. Kaur 